\title{
A Bayesian consistent dual ensemble Kalman filter for state-parameter estimation in subsurface hydrology
}

\author{
Boujemaa Ait-El-Fquih ${ }^{1}$, Mohamad El Gharamti ${ }^{1,2}$, and Ibrahim Hoteit ${ }^{1}$ \\ ${ }^{1}$ Department of Earth Sciences and Engineering, King Abdullah University of Science and Technology (KAUST), \\ 23955-6900 Thuwal, Kingdom of Saudi Arabia \\ ${ }^{2}$ Mohn-Sverdrup Center for Global Ocean Studies and Operational Oceanography, Nansen Environmental and Remote \\ Sensing Center (NERSC), 5006 Bergen, Norway
}

Correspondence to: Ibrahim Hoteit (ibrahim.hoteit@kaust.edu.sa)

Received: 16 December 2015 - Published in Hydrol. Earth Syst. Sci. Discuss.: 1 February 2016

Revised: 29 June 2016 - Accepted: 10 July 2016 - Published: 12 August 2016

\begin{abstract}
Ensemble Kalman filtering (EnKF) is an efficient approach to addressing uncertainties in subsurface groundwater models. The EnKF sequentially integrates field data into simulation models to obtain a better characterization of the model's state and parameters. These are generally estimated following joint and dual filtering strategies, in which, at each assimilation cycle, a forecast step by the model is followed by an update step with incoming observations. The joint EnKF directly updates the augmented state-parameter vector, whereas the dual EnKF empirically employs two separate filters, first estimating the parameters and then estimating the state based on the updated parameters. To develop a Bayesian consistent dual approach and improve the stateparameter estimates and their consistency, we propose in this paper a one-step-ahead (OSA) smoothing formulation of the state-parameter Bayesian filtering problem from which we derive a new dual-type EnKF, the dual EnKF ${ }_{\mathrm{OSA}}$. Compared with the standard dual EnKF, it imposes a new update step to the state, which is shown to enhance the performance of the dual approach with almost no increase in the computational cost. Numerical experiments are conducted with a twodimensional (2-D) synthetic groundwater aquifer model to investigate the performance and robustness of the proposed dual $\mathrm{EnKF}_{\mathrm{OSA}}$, and to evaluate its results against those of the joint and dual EnKFs. The proposed scheme is able to successfully recover both the hydraulic head and the aquifer conductivity, providing further reliable estimates of their uncertainties. Furthermore, it is found to be more robust to different assimilation settings, such as the spatial and temporal distribution of the observations, and the level of noise in the
\end{abstract}

data. Based on our experimental setups, it yields up to $25 \%$ more accurate state and parameter estimations than the joint and dual approaches.

\section{Introduction}

In modern hydrology research, uncertainty quantification studies have focused on field applications, including surface and subsurface water flow, contaminant transport, and reservoir engineering. The motivations behind these studies were driven by the uncertain and stochastic nature of hydrological systems. For instance, surface rainfall-runoff models that account for soil moisture and streamflows are subject to many uncertain parameters such as free- and tension water storage content, water depletion rates, and melting threshold temperatures (Samuel et al., 2014). Groundwater flow models, on the other hand, depend on our knowledge of spatially variable aquifer properties, such as porosity and hydraulic conductivity, which are often poorly known (Chen and Zhang, 2006; Hendricks Franssen and Kinzelbach, 2008). In addition, contaminant transport models that investigate the migration of pollutants in subsurface aquifers are quite sensitive to reaction parameters, e.g., sorption rates, radioactive decay, and biodegradation (Gharamti and Hoteit, 2014; Gharamti et al., 2014b). To this end, it is important to study the variability of hydrological parameters and reduce their associated uncertainties in order to obtain reliable simulations. To achieve this goal, hydrologists have resorted to various inverse and Monte Carlo-based statistical techniques with the standard 
procedure of pinpointing parameter values that, when integrated with the simulation models, allow some systemresponse variables (e.g., hydraulic head, solute concentration) to fit given observations (Vrugt et al., 2003; Valstar et al., 2004; Alcolea et al., 2006; Feyen et al., 2007; Hendricks Franssen and Kinzelbach, 2009; Zhou et al., 2014). Recently, sequential data assimilation techniques, such as the particle filter (PF), have been proposed to handle any type of statistical distribution, Gaussian or not, to properly deal with strongly nonlinear systems (Chang et al., 2012). The PF may require, however, a prohibitive number of particles (and thus model runs) to accurately sample the distribution of the state and parameters, making this scheme computationally intensive for large-scale hydrological applications (Doucet et al., 2001; Moradkhani et al., 2005a; Hoteit et al., 2008; Montzka et al., 2011). This problem has been partially addressed by the popular ensemble Kalman filter (EnKF), which further provides robustness, efficiency, and non-intrusive formulation (Reichle et al., 2002; Vrugt et al., 2006; Zhou et al., 2011; Gharamti et al., 2013; Panzeri et al., 2014; Crestani et al., 2013; McMillam et al., 2013; Erdal and Cirpka, 2016) to tackle the state-parameter estimation problem.

The EnKF is a filtering technique that is relatively simple to implement, even with complex nonlinear models, requiring only an observation operator that maps the state variables from the model space into the observation space. Compared with traditional inverse and direct optimization techniques, which are generally based on least-squares-like formulations, the EnKF has the advantage of being able to account for model errors that are present not only in the uncertain parameters but also in the external forcings (Hendricks Franssen and Kinzelbach, 2008). Because of its sequential formulation, the EnKF does not require one to store all past information about the states and parameters, leading to consequent savings in computational cost (McLaughlin, 2002; Gharamti et al., 2014b).

The EnKF is widely used in surface and subsurface hydrological studies to tackle state-parameter estimation problems (Zhou et al., 2014; Panzeri et al., 2014). Two approaches are usually considered based on the joint and the dual estimation strategies. The standard joint approach concurrently estimates the state and the parameters by augmenting (in the same vector) the state variables with the unknown parameters, that do not vary in time. The parameters could also be set to follow an artificial evolution (random walk) before they get updated with incoming observations (Wan et al., 1999). One of the early applications of the joint EnKF to subsurface groundwater flow models was presented by Chen and Zhang (2006). In their study, a conceptual subsurface flow system was considered and ensemble filtering was performed to estimate the transient pressure field alongside the hydraulic conductivity. In a reservoir engineering application, Nævdal et al. (2005) considered a two-dimensional (2-D) North Sea field model and considered the joint estimation problem of the dynamic pressure and saturation on top of the static permeability field. Groundwater contamination problems were also tackled using the joint EnKF (e.g., Li et al., 2012; Gharamti and Hoteit, 2014), in which the hydraulic head, contaminant concentration, and spatially variable permeability and porosity parameters were estimated for coupled groundwater flow and contaminant transport systems.

Several studies argued that the joint EnKF may suffer from important inconsistencies between the estimated state and parameters that could degrade the filter performance, especially with large-dimensional and strongly nonlinear systems (e.g., Moradkhani et al., 2005b; Chen and Zhang, 2006; Wen and Chen, 2007). One classical approach that has been proposed to tackle this issue is the so-called dual filter, which separately updates the state and parameters using two interactive EnKFs, one acting on the state and the other on the parameters (Moradkhani et al., 2005b). The dual EnKF has been applied to streamflow forecasting problems using rainfall-runoff models (e.g., Lü et al., 2013; Samuel et al., 2014), subsurface contaminant (e.g., Tian et al., 2008; Lü et al., 2011; Gharamti et al., 2014b), and compositional flow models (e.g., Phale and Oliver, 2011; Gharamti et al., 2014a), to cite but a few. Gharamti et al. (2014a) concluded that the dual scheme provides more accurate state and parameter estimations than the joint scheme when implemented with large enough ensembles. In terms of complexity, however, the dual scheme requires integrating the filter ensemble twice with the numerical model at every assimilation cycle, and is therefore computationally more demanding. In related works, Gharamti et al. (2013) extended the dual filtering scheme to tackle the state estimation problem of oneway coupled models, and to the framework of hybrid-EnKF (Gharamti et al., 2014b).

The dual filter has been basically introduced as a heuristic scheme and is not consistent with the Bayesian filtering framework (Hendricks Franssen and Kinzelbach, 2008). A first attempt to build a Bayesian consistent dual-like filter was recently proposed by Gharamti et al. (2015) in which a new joint EnKF scheme was derived following the one-stepahead (OSA) smoothing formulation of the Bayesian filtering problem. The new joint scheme reverses the order of the measurement-update and the forecast (or time) update, leading to two Kalman-like update steps based on the current observations: one for state smoothing and one for parameters updating.

Motivated by the promising results of Gharamti et al. (2015), we follow here the same OSA smoothing formulation to derive a new dual EnKF, which we refer to as the dual EnKF $F_{O S A}$ hereafter, generalizing the joint scheme of Gharamti et al. (2015) and, in particular, the standard dual EnKF. Our goal is to derive a new dual EnKF-like algorithm that retains the separate formulation of the state and parameters update steps, within a fully Bayesian framework. The new dual-type filter relaxes the assumption of independence between the state and its observation conditionally on the previous state and parameters, which have been im- 
posed by Gharamti et al. (2015). Exploiting the conditional dependency between the state and its observation leads to one more Kalman-like update of the state, generalizing the joint scheme of Gharamti et al. (2015), at practically no increase in the computational cost. Likewise, the new dual filtering scheme is not only more general than the standard dual scheme, but also explicitly derives the conditions under which the (heuristic) steps of the standard dual EnKF can be derived within a Bayesian framework. Synthetic numerical experiments based on a groundwater flow model and estimating the hydraulic head and the conductivity parameter field, are conducted to assess the performance of the proposed dual $\mathrm{EnKF}_{\mathrm{OSA}}$ and to compare its results against those of the joint and the dual EnKFs, which we consider as references to evaluate the behavior of the dual EnKF OSA. The numerical results suggest that the proposed scheme is beneficial in terms of estimation accuracy compared to the two standard joint and dual schemes, and is more robust to various experimental settings and observational scenarios.

The rest of the paper is organized as follows. Section 2 reviews the standard joint and dual EnKF strategies. The dual EnKF $_{\text {OSA }}$ is derived in Sect. 3 and its relation with the joint and dual EnKFs is discussed. Section 4 presents a conceptual groundwater flow model and outlines the experimental setup. Numerical results are presented and discussed in Sect. 5. Conclusions are offered in Sect. 6, followed by an Appendix.

\section{Joint and dual ensemble Kalman filtering}

\subsection{Problem formulation}

Consider a discrete-time state-parameter dynamical system:

$$
\left\{\begin{array}{l}
\mathbf{x}_{n}=\mathcal{M}_{n-1}\left(\mathbf{x}_{n-1}, \theta\right)+\eta_{n-1} \\
\mathbf{y}_{n}=\mathbf{H}_{n} \mathbf{x}_{n}+\varepsilon_{n}
\end{array},\right.
$$

where $\mathbf{x}_{n} \in \mathbb{R}^{N_{x}}$ and $\mathbf{y}_{n} \in \mathbb{R}^{N_{y}}$ denote the system state and the observation at time $t_{n}$ of dimensions $N_{x}$ and $N_{y}$, respectively, and $\theta \in \mathbb{R}^{N_{\theta}}$ is the parameter vector of dimension $N_{\theta} \cdot \mathcal{M}_{n}$ is a nonlinear operator integrating the system state from time $t_{n}$ to $t_{n+1}$, and the observational operator at time $t_{n}, \mathbf{H}_{n}$, is assumed to be linear for simplicity; the proposed scheme can be easily extended to the nonlinear case ${ }^{1}$. The model process noise, $\eta=\left\{\eta_{n}\right\}_{n \in \mathbb{N}}$, and the observation process noise, $\varepsilon=\left\{\varepsilon_{n}\right\}_{n \in \mathbb{N}}$, are assumed to be independent, jointly independent, and independent of $\mathbf{x}_{0}$ and $\theta$. Furthermore, let $\eta_{n}$ and $\varepsilon_{n}$ be Gaussian with zero means and covariances $\mathbf{Q}_{n}$ and $\mathbf{R}_{n}$, respectively. Throughout the paper, $\mathbf{y}_{0: n} \stackrel{\text { def }}{=}\left\{\mathbf{y}_{0}, \mathbf{y}_{1}, \cdots, \mathbf{y}_{n}\right\}$ and $p\left(\mathbf{x}_{n}\right)$ and $p\left(\mathbf{x}_{n} \mid \mathbf{y}_{0: l}\right)$ stand for the prior probability density function (pdf) of $\mathbf{x}_{n}$ and the posterior pdf of $\mathbf{x}_{n}$ given $\mathbf{y}_{0: l}$, respectively. All other pdf's used are defined in a similar way.

\footnotetext{
${ }^{1}$ The term $\mathbf{H}_{n} \mathbf{x}_{n}^{\mathrm{f},(m)}$ is replaced by $\mathcal{H}_{n}\left(\mathbf{x}_{n}^{\mathrm{f},(m)}\right)$ in Eq. (26), and $\mathbf{H}_{n} \xi_{n}^{(m)}$ is replaced by $\mathcal{H}_{n}\left(\xi_{n}^{(m)}\right)$ in Eq. (34).
}

We focus on the state-parameter filtering problem, i.e., the estimation at each time, $t_{n}$, of the state, $\mathbf{x}_{n}$, as well as the parameters vector, $\boldsymbol{\theta}$, from the history of the observations, $\mathbf{y}_{0: n}$. The standard solution of this problem is the a posteriori mean (AM):

$$
\begin{aligned}
& \mathbb{E}_{p\left(x_{n} \mid y_{0: n}\right)}\left[\mathbf{x}_{n}\right]=\int \mathbf{x}_{n} p\left(\mathbf{x}_{n}, \theta \mid \mathbf{y}_{0: n}\right) \mathrm{d} \mathbf{x}_{n} \mathrm{~d} \theta, \\
& \mathbb{E}_{p\left(\theta \mid y_{0: n}\right)}[\theta]=\int \theta p\left(\mathbf{x}_{n}, \theta \mid \mathbf{y}_{0: n}\right) \mathrm{d} \mathbf{x}_{n} \mathrm{~d} \theta,
\end{aligned}
$$

which minimizes the a posteriori mean square error. In practice, analytical computation of Eqs. (2) and (3) is not feasible, mainly due to the nonlinear character of the system. The joint and dual EnKFs have been introduced as efficient schemes to compute approximations of Eqs. (2) and (3). These algorithms are reviewed in the next section.

\subsection{The joint and dual EnKFs}

\subsubsection{The joint EnKF}

The key idea behind the joint EnKF is to transform the stateparameter system (Eq. 1) into a classical state-space system based on the augmented state, $\mathbf{z}_{n}=\left[\mathbf{x}_{n}^{T} \theta^{T}\right]^{T}$, on which the classical EnKF can be directly applied. The new augmented state-space system can be written as

$$
\left\{\begin{array}{l}
\mathbf{z}_{n}=\widetilde{\mathcal{M}}_{n-1}\left(\mathbf{z}_{n-1}\right)+\widetilde{\eta}_{n-1} \\
\mathbf{y}_{n}=\widetilde{\mathbf{H}}_{n} \mathbf{z}_{n}+\varepsilon_{n}
\end{array}\right.
$$

where $\widetilde{\mathcal{M}}_{n-1}\left(\mathbf{z}_{n-1}\right)=\left[\begin{array}{c}\mathcal{M}_{n-1}\left(\mathbf{z}_{n-1}\right) \\ \theta\end{array}\right], \widetilde{\eta}_{n-1}=\left[\begin{array}{ll}\eta_{n-1}^{T} & \mathbf{0}\end{array}\right]^{T}$, $\widetilde{\mathbf{H}}_{n}=\left[\mathbf{H}_{n} \mathbf{0}\right]$, with $\mathbf{0}$ a zero matrix with appropriate dimensions. $\mathbf{x}_{n}^{\mathrm{f},(m)}, \mathbf{x}_{n}^{\mathrm{a},(m)}$, and $\mathbf{x}_{n}^{\mathrm{s},(m)}$ respectively denote the $m$ th forecast, analysis, and (OSA) smoothing member of the state, $\mathbf{x}_{n}$, while $\theta_{\mid n}^{(m)}$ denotes the $m$ th sample of the parameters posterior pdf, $p\left(\theta \mid \mathbf{y}_{0: n}\right)$. Since the parameters are static (i.e., time-independent), $\theta_{\mid n}^{(m)}, n=1,2, \cdots$, could be considered as analysis, forecast, or smoothing members.

Starting at time $t_{n-1}$ from an analysis ensemble of size $N_{\mathrm{e}}$, $\left\{\mathbf{x}_{n-1}^{\mathrm{a},(m)}, \theta_{\mid n-1}^{(m)}\right\}_{m=1}^{N_{\mathrm{e}}}$ sampled from $p\left(\mathbf{z}_{n-1} \mid \mathbf{y}_{0: n-1}\right)$, the EnKF uses the augmented state model (1st equation of Eq. 4) to compute the forecast ensemble, $\left\{\mathbf{x}_{n}^{\mathrm{f},(m)}, \theta_{\mid n-1}^{(m)}\right\}_{m=1}^{N_{\mathrm{e}}}$, approximating $p\left(\mathbf{z}_{n} \mid \mathbf{y}_{0: n-1}\right)$. The observation model ( 2 nd equation of Eq. 4) is then used to obtain the analysis ensemble, $\left\{\mathbf{x}_{n}^{\mathrm{a},(m)}\right.$, $\left.\theta_{\mid n}^{(m)}\right\}_{m=1}^{N_{\mathrm{e}}}$, at time $t_{n}$. Let, for an ensemble $\left\{\mathbf{r}^{(m)}\right\}_{m=1}^{N_{\mathrm{e}}}, \hat{\mathbf{r}} \mathrm{de}-$ note its empirical mean and $\mathbf{S}_{r}$ a matrix with $N_{\mathrm{e}}$-columns whose $m$ th column is defined as $\left(\mathbf{r}^{(m)}-\hat{\mathbf{r}}\right)$. The joint EnKF steps can be summarized as follows:

- Forecast step: the parameters vector members, $\theta_{\mid n-1}^{(m)}$, are kept invariant, while the state vector members, $\mathbf{x}_{n-1}^{\mathrm{a},(m)}$ are integrated in time through the dynamical model as 


$$
\begin{aligned}
\mathbf{x}_{n}^{\mathrm{f},(m)} & =\mathcal{M}_{n-1}\left(\mathbf{x}_{n-1}^{\mathrm{a},(m)}, \theta_{\mid n-1}^{(m)}\right)+\eta_{n-1}^{(m)} ; \eta_{n-1}^{(m)} \\
& \sim \mathcal{N}\left(\mathbf{0}, \mathbf{Q}_{n-1}\right) .
\end{aligned}
$$

The state forecast estimate, which is the mean of $p\left(\mathbf{x}_{n} \mid \mathbf{y}_{0: n-1}\right)$ (i.e., $\left.\mathbb{E}_{p\left(x_{n} \mid y_{0: n-1}\right)}\left[\mathbf{x}_{n}\right]\right)$, is taken as the empirical mean of the forecast ensemble, $\hat{\mathbf{x}}_{n}^{\mathrm{f}}$. The associated forecast error covariance is estimated as $\mathbf{P}_{x_{n}^{\mathrm{f}}}=\left(N_{\mathrm{e}}-1\right)^{-1} \mathbf{S}_{x_{n}^{\mathrm{f}}} \mathbf{S}_{x_{n}^{\mathrm{f}}}^{T}$.

- Analysis step: once a new observation is available, all members, $\mathbf{x}_{n}^{\mathrm{f},(m)}$ and $\theta_{\mid n-1}^{(m)}$, are updated as in the Kalman filter (KF):

$$
\begin{aligned}
& \mathbf{y}_{n}^{\mathrm{f},(m)}=\mathbf{H}_{n} \mathbf{x}_{n}^{\mathrm{f},(m)}+\varepsilon_{n}^{(m)} ; \varepsilon_{n}^{(m)} \sim \mathcal{N}\left(\mathbf{0}, \mathbf{R}_{n}\right), \\
& \mathbf{x}_{n}^{\mathrm{a},(m)}=\mathbf{x}_{n}^{\mathrm{f},(m)}+\mathbf{P}_{x_{n}^{\mathrm{f}}, y_{n}^{\mathrm{f}}} \underbrace{\mathbf{P}_{y_{n}^{\mathrm{f}}}^{-1}\left(\mathbf{y}_{n}-\mathbf{y}_{n}^{\mathrm{f},(m)}\right)}_{\mu_{n}^{(m)}}, \\
& \theta_{\mid n}^{(m)}=\theta_{\mid n-1}^{(m)}+\mathbf{P}_{\theta_{\mid n-1}, y_{n}^{\mathrm{f}}} \cdot \mu_{n}^{(m)} .
\end{aligned}
$$

The (cross-)covariances in Eqs. (7) and (8) are practically evaluated from the ensembles as

$$
\begin{aligned}
& \mathbf{P}_{x_{n}^{\mathrm{f}}, y_{n}^{\mathrm{f}}}=\left(N_{\mathrm{e}}-1\right)^{-1} \mathbf{S}_{x_{n}^{\mathrm{f}}} \mathbf{S}_{y_{n}^{\mathrm{f}}}^{T}, \\
& \mathbf{P}_{y_{n}^{\mathrm{f}}}=\left(N_{\mathrm{e}}-1\right)^{-1} \mathbf{S}_{y_{n}^{\mathrm{f}}} \mathbf{S}_{y_{n}^{\mathrm{f}}}^{T}, \\
& \mathbf{P}_{\theta_{\mid n-1}, y_{n}^{\mathrm{f}}}=\left(N_{\mathrm{e}}-1\right)^{-1} \mathbf{S}_{\theta_{\mid n-1}} \mathbf{S}_{y_{n}^{\mathrm{f}}}^{T} .
\end{aligned}
$$

The analysis estimates, Eqs. (2) and (3), and their error covariances, can thus be approximated by the analysis ensemble means, $\hat{\mathbf{x}}_{n}^{\mathrm{a}}$ and $\hat{\theta}_{\mid n}$, and covariances $\mathbf{P}_{x_{n}^{\mathrm{a}}}=\left(N_{\mathrm{e}}-1\right)^{-1} \mathbf{S}_{x_{n}^{\mathrm{a}}} \mathbf{S}_{x_{n}^{\mathrm{a}}}^{T}$ and $\mathbf{P}_{\theta_{\mid n}}=\left(N_{\mathrm{e}}-1\right)^{-1} \mathbf{S}_{\theta \mid n} \mathbf{S}_{\theta_{\mid n}}^{T}$, respectively. Note that $\mathbf{P}_{x_{n}^{\mathrm{f}}, y_{n}^{\mathrm{f}}} \mathbf{P}_{y_{n}^{\mathrm{f}}}^{-1}$ in Eq. (7) represents the Kalman Gain, $\mathbf{P}_{x_{n}^{\mathrm{f}}} \mathbf{H}_{n}^{T}\left[\mathbf{H}_{n} \mathbf{P}_{x_{n}^{\mathrm{f}}} \mathbf{H}_{n}^{T}+\mathbf{R}_{n}\right]^{-1}$. This statistical formulation of the Kalman gain offers more flexibility to deal with nonlinear observational operators (Moradkhani et al., 2005b).

\subsubsection{The dual EnKF}

In contrast with the joint EnKF, the dual EnKF is empirically designed following a conditional estimation strategy, operating as a succession of two EnKF-like filters. First, a (parameter) filter is applied to compute $\left\{\theta_{\mid n}^{(m)}\right\}_{m=1}^{N_{\mathrm{e}}}$ from $\left\{\mathbf{x}_{n-1}^{\mathrm{a},(m)}\right.$, $\left.\theta_{\mid n-1}^{(m)}\right\}_{m=1}^{N_{\mathrm{e}}}$ exactly as in the joint EnKF:

- Forecast step: the parameters ensemble, $\left\{\theta_{\mid n-1}^{(m)}\right\}_{m=1}^{N_{\mathrm{e}}}$, is kept invariant, while the state samples are integrated in time as in Eq. (5) to compute the forecast ensemble, $\left\{\mathbf{x}_{n}^{\mathrm{f},(m)}\right\}_{m=1}^{N_{\mathrm{e}}}$.
- Analysis step: as in Eq. (6), the observation forecast ensemble $\left\{\mathbf{y}_{n}^{\mathrm{f},(m)}\right\}_{m=1}^{N_{\mathrm{e}}}$ is computed from $\left\{\mathbf{x}_{n}^{\mathrm{f},(m)}\right\}_{m=1}^{N_{\mathrm{e}}}$. This is then used to update the parameters ensemble, $\left\{\theta_{\mid n}^{(m)}\right\}_{m=1}^{N_{\mathrm{e}}}$, following Eq. (8).

Another (state) filter is then applied to compute $\left\{\mathbf{x}_{n}^{\mathrm{a},(m)}\right\}_{m=1}^{N_{\mathrm{e}}}$ from $\left\{\mathbf{x}_{n-1}^{\mathrm{a},(m)}\right\}_{m=1}^{N_{\mathrm{e}}}$ as well as the new parameter ensemble, $\left\{\theta_{\mid n}^{(m)}\right\}_{m=1}^{N_{\mathrm{e}}}$, again in two steps that can be summarized as follows.

- Forecast step: each member, $\mathbf{x}_{n-1}^{\mathrm{a},(m)}$, is propagated in time with the dynamical model using the updated parameters ensemble:

$$
\widetilde{\mathbf{x}}_{n}^{\mathrm{f},(m)}=\mathcal{M}_{n-1}\left(\mathbf{x}_{n-1}^{\mathrm{a},(m)}, \theta_{\mid n}^{(m)}\right) .
$$

- Analysis step: as in the parameter filter, $\left\{\widetilde{\mathbf{y}}_{n}^{\mathrm{f},(m)}\right\}_{m=1}^{N_{\mathrm{e}}}$ is computed from $\left\{\widetilde{\mathbf{x}}_{n}^{\mathrm{f},(m)}\right\}_{m=1}^{N_{\mathrm{e}}}$ using Eq. (6), which finally yields $\left\{\mathbf{x}_{n}^{\mathrm{a},(m)}\right\}_{m=1}^{N_{\mathrm{e}}}$ as in Eq. (7).

To better understand how the dual EnKF differs from the joint EnKF, we focus on how the analysis members at time $t_{n}$, namely, $\mathbf{x}_{n}^{\mathrm{a},(m)}$ and $\theta_{\mid n}^{(m)}$, are obtained starting from their counterparts at previous time, $\mathbf{x}_{n-1}^{\mathrm{a},(m)}$ and $\theta_{\left.\right|_{n-1}}^{(m)}$. The parameters members, $\theta_{\mid n}^{(m)}$, are computed based on the same equation (Eq. 8) in both algorithms. For the state members, $\mathbf{x}_{n}^{\mathrm{a},(m)}$, we have

$$
\begin{aligned}
& \mathbf{x}_{n}^{\mathrm{a},(m)} \stackrel{\text { joint }}{=}{ }^{\text {EnKF }} \mathcal{M}_{n-1}\left(\mathbf{x}_{n-1}^{\mathrm{a},(m)}, \theta_{\mid n-1}^{(m)}\right)+\mathbf{P}_{x_{n}^{\mathrm{f}}, y_{n}^{\mathrm{f}}} \\
& \underbrace{\mathbf{P}_{y_{n}^{\mathrm{f}}}^{-1}\left(\mathbf{y}_{n}-\mathbf{y}_{n}^{\mathrm{f},(m)}\right)}_{\mu_{n}^{(m)}},
\end{aligned}
$$

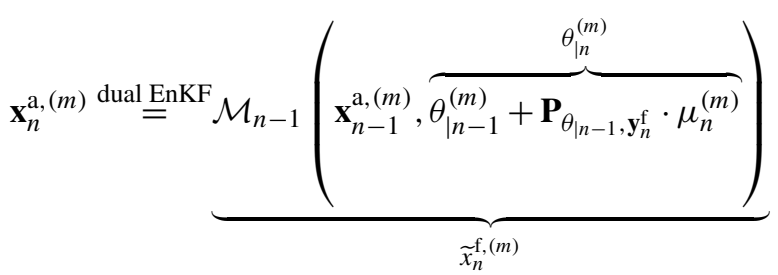

$$
\begin{aligned}
& +\mathbf{P}_{\widetilde{x}_{n}^{\mathrm{f}}, \widetilde{\mathbf{Y}}_{n}^{\mathrm{f}}} \underbrace{\mathbf{P}_{\widetilde{y}_{n}^{f}}^{-1}\left(\mathbf{y}_{n}-\widetilde{\mathbf{y}}_{n}^{\mathrm{f},(m)}\right)}_{\widetilde{\mu}_{n}^{(m)}} .
\end{aligned}
$$

For simplicity, we ignore here the process noise term, $\eta_{n}$, which is commonly applied in geophysical applications. As one can see, the joint EnKF updates the state members using one Kalman-like correction (term of $\mu_{n}^{(m)}$ in Eq. (13)), whereas the dual EnKF applies two Kalman-like corrections. More specifically, the dual EnKF updates first the parameters members, $\theta_{\mid n-1}^{(m)}$, as in the joint EnKF, leading to $\theta_{\mid n}^{(m)}$; these are then used to propagate $\mathbf{x}_{n-1}^{\mathrm{a},(m)}$, with the model to provide 
the "forecast" members $\widetilde{\mathbf{x}}_{n}^{\mathbf{f},(m)}$. The $\widetilde{\mathbf{x}}_{n}^{\mathbf{f},(m)}$ are finally updated using a Kalman-like correction (term of $\widetilde{\mu}_{n}^{(m)}$ in Eq. 14), to obtain the analysis members $\mathbf{x}_{n}^{\mathrm{a},(m)}$. Such a separation of the update steps is expected to provide more consistent estimates of the parameters. The dual update framework was indeed shown to provide better performances than the joint EnKF, at the cost of increased computational burden (see for instance, Moradkhani et al., 2005b; Samuel et al., 2014; Gharamti et al., 2014a).

\subsubsection{Probabilistic formulation}

Following a probabilistic formulation, the augmented state system (Eq. 4) can be viewed as a continuous state hidden Markov chain with transition density,

$$
\begin{aligned}
p\left(\mathbf{z}_{n} \mid \mathbf{z}_{n-1}\right) & =p\left(\mathbf{x}_{n} \mid \mathbf{x}_{n-1}, \theta\right) p(\theta \mid \theta) \\
& =\mathcal{N}_{x_{n}}\left(\mathcal{M}_{n-1}\left(\mathbf{x}_{n-1}, \theta\right), \mathbf{Q}_{n-1}\right),
\end{aligned}
$$

and likelihood,

$p\left(\mathbf{y}_{n} \mid \mathbf{z}_{n}\right)=p\left(\mathbf{y}_{n} \mid \mathbf{x}_{n}\right)=\mathcal{N}_{y_{n}}\left(\mathbf{H}_{n} \mathbf{x}_{n}, \mathbf{R}_{n}\right)$,

where $\mathcal{N}_{v}(\mathbf{m}, \mathbf{C})$ represents a Gaussian pdf of argument $\mathbf{v}$ and parameters $(\mathbf{m}, \mathbf{C})$.

One can then easily verify that the joint EnKF can be derived from a direct application of two classical results of random sampling (Properties 1 and 2 in Appendix A) on the following classical generic formulas:

$$
\begin{aligned}
& p\left(\mathbf{z}_{n} \mid \mathbf{y}_{0: n-1}\right)=\int p\left(\mathbf{x}_{n} \mid \mathbf{x}_{n-1}, \theta\right) p\left(\mathbf{z}_{n-1} \mid \mathbf{y}_{0: n-1}\right) \mathrm{d} \mathbf{x}_{n-1}, \\
& p\left(\mathbf{y}_{n} \mid \mathbf{y}_{0: n-1}\right)=\int p\left(\mathbf{y}_{n} \mid \mathbf{x}_{n}\right) p\left(\mathbf{x}_{n} \mid \mathbf{y}_{0: n-1}\right) \mathrm{d} \mathbf{x}_{n}, \\
& p\left(\mathbf{z}_{n} \mid \mathbf{y}_{0: n}\right)=\frac{p\left(\mathbf{z}_{n}, \mathbf{y}_{n} \mid \mathbf{y}_{0: n-1}\right)}{p\left(\mathbf{y}_{n} \mid \mathbf{y}_{0: n-1}\right)}
\end{aligned}
$$

Equation (17) refers to a Markovian step (or time-update step) and uses the transition pdf, $p\left(\mathbf{x}_{n} \mid \mathbf{x}_{n-1}, \theta\right)$, of the Markov chain, $\left\{\mathbf{z}_{n}\right\}_{n}$, to compute the forecast pdf of $\mathbf{z}_{n}$ from the previous analysis pdf. Equation (19) refers to a Bayesian step (or measurement-update step) since it uses the Bayes' rule to update the forecast pdf of $\mathbf{z}_{n}$ using the current observation $\mathbf{y}_{n}$. Thus, establishing the link between the joint EnKF and Eqs. (17)-(19), one can show that Property 1 and Eq. (17) lead to the forecast ensemble of the state (Eq. 5). Property 1 and Eq. (18) lead to the forecast ensemble of the observations (Eq. 6). Property 2 and Eq. (19) then provide the analysis ensemble of the state (Eq. 7) and the parameters (Eq. 8).

Regarding the dual EnKF, the forecast ensemble of the state and observations in the parameter filter can be obtained following the same process as in the joint EnKF. This is followed by the computation of the analysis ensemble of the parameters using Property 2 and

$p\left(\theta \mid \mathbf{y}_{0: n}\right)=\frac{p\left(\theta, \mathbf{y}_{n} \mid \mathbf{y}_{0: n-1}\right)}{p\left(\mathbf{y}_{n} \mid \mathbf{y}_{0: n-1}\right)}$.
However, in the state filter, the ensemble, $\left\{\tilde{\mathbf{x}}_{n}^{\mathrm{f},(m)}\right\}_{m=1}^{N_{\mathrm{e}}}$, obtained via Eq. (12) in the forecast step does not represent the forecast pdf, $p\left(\mathbf{x}_{n} \mid \mathbf{y}_{0: n-1}\right)$, since Eq. (12) involves $\theta_{\mid n}^{(m)}$ rather than $\theta_{\mid n-1}^{(m)}$. Accordingly, the dual EnKF is basically a heuristic algorithm in spite of its proven performance.

\section{One-step-ahead smoothing-based dual EnKF (dual EnKF $_{\text {OSA }}$ )}

The classical (time-update, measurement-update) path (Eqs. 17-19) to compute the analysis pdf $p\left(\mathbf{z}_{n} \mid \mathbf{y}_{0: n}\right)$ from $p\left(\mathbf{z}_{n-1} \mid \mathbf{y}_{0: n-1}\right)$ is not the only possible one. One may indeed reverse the order of the time- and measurement-update steps by involving the OSA smoothing pdf, $p\left(\mathbf{z}_{n-1} \mid \mathbf{y}_{0: n}\right)$, between two successive analysis pdf's: $p\left(\mathbf{z}_{n-1} \mid \mathbf{y}_{0: n-1}\right)$ and $p\left(\mathbf{z}_{n} \mid \mathbf{y}_{0: n}\right)$. Desbouvries et al. (2011) considered the OSA smoothing-based filtering problem in low-dimensional statespace systems to derive a class of KF- and PF-like algorithms for filtering the state. The more recent work of Lee and Farmer (2014) proposed a number of algorithms to estimate both the system state and the model noise based on a similar strategy. In the context of large-dimensional stateparameters filtering, we show in this section that this leads to a new fully Bayesian consistent dual-like filtering scheme, the dual EnKF $\mathrm{OSA}_{\mathrm{O} A}$, which, compared to the standard dual EnKF, not only introduces another Kalman-like update of the state but also involves a (new) smoothing step that constraints the state with the future observation. Exploiting the future observation should be particularly beneficial in the context of the EnKF as it includes more information in the estimation process that may help mitigating for the suboptimal character of the EnKF-like methods, being formulated under a linear Gaussian framework, and usually implemented with limited ensembles and crude approximate noise statistics.

\subsection{The one-step-ahead smoothing-based dual filtering algorithm}

The analysis pdf, $p\left(\mathbf{x}_{n}, \theta \mid \mathbf{y}_{0: n}\right)$, can be computed from $p\left(\mathbf{x}_{n-1}, \theta \mid \mathbf{y}_{0: n-1}\right)$ in two steps:

- Smoothing step: the one-step-ahead smoothing pdf, $p\left(\mathbf{x}_{n-1}, \theta \mid \mathbf{y}_{0: n}\right)$, is first computed as

$$
\begin{gathered}
p\left(\mathbf{x}_{n-1}, \theta \mid \mathbf{y}_{0: n}\right) \propto p\left(\mathbf{y}_{n} \mid \mathbf{x}_{n-1}, \theta, \mathbf{y}_{0: n-1}\right) \\
p\left(\mathbf{x}_{n-1}, \theta \mid \mathbf{y}_{0: n-1}\right),
\end{gathered}
$$

with,

$$
\begin{aligned}
& p\left(\mathbf{y}_{n} \mid \mathbf{x}_{n-1}, \theta, \mathbf{y}_{0: n-1}\right)=\int p\left(\mathbf{y}_{n} \mid \mathbf{x}_{n}, \mathbf{x}_{n-1}, \theta, \mathbf{y}_{0: n-1}\right) \\
& p\left(\mathbf{x}_{n} \mid \mathbf{x}_{n-1}, \theta, \mathbf{y}_{0: n-1}\right) \mathrm{d} \mathbf{x}_{n},=\int p\left(\mathbf{y}_{n} \mid \mathbf{x}_{n}\right) \\
& p\left(\mathbf{x}_{n} \mid \mathbf{x}_{n-1}, \theta\right) \mathrm{d} \mathbf{x}_{n} .
\end{aligned}
$$


Equation (22) is derived from the fact that in the stateparameter model (Eq. 1), the observation noise, $\varepsilon_{n}$, and the model noise, $\eta_{n-1}$, are independent of $\left(\mathbf{x}_{n-1}, \theta\right)$ and past observations $\mathbf{y}_{0: n-1}$.

The smoothing step (Eq. 21) is indeed a measurementupdate step since, given $\mathbf{y}_{0: n-1}$, Eq. (21) translates the computation of the posterior, $p\left(\mathbf{x}_{n-1}, \theta \mid \mathbf{y}_{n}\right)$, as a normalized product of the prior, $p\left(\mathbf{x}_{n-1}, \theta\right)$, and the likelihood, $p\left(\mathbf{y}_{n} \mid \mathbf{x}_{n-1}, \theta\right)$ (note from Eq. (22) that $p\left(\mathbf{y}_{n} \mid \mathbf{x}_{n-1}\right.$, $\left.\left.\theta, \mathbf{y}_{0: n-1}\right)=p\left(\mathbf{y}_{n} \mid \mathbf{x}_{n-1}, \theta\right)\right)$.

- Forecast step: the smoothing pdf at $t_{n-1}$ is then used to compute the current analysis pdf, $p\left(\mathbf{x}_{n}, \theta \mid \mathbf{y}_{0: n}\right)$, as

$$
\begin{aligned}
p\left(\mathbf{x}_{n}, \theta \mid \mathbf{y}_{0: n}\right)= & \int p\left(\mathbf{x}_{n} \mid \mathbf{x}_{n-1}, \theta, \mathbf{y}_{0: n}\right) \\
& p\left(\mathbf{x}_{n-1}, \theta \mid \mathbf{y}_{0: n}\right) \mathrm{d} \mathbf{x}_{n-1},
\end{aligned}
$$

with,

$p\left(\mathbf{x}_{n} \mid \mathbf{x}_{n-1}, \theta, \mathbf{y}_{0: n}\right) \propto p\left(\mathbf{y}_{n} \mid \mathbf{x}_{n}\right) p\left(\mathbf{x}_{n} \mid \mathbf{x}_{n-1}, \theta\right)$,

which, in turn, arises from the fact that $\varepsilon_{n}$ and $\eta_{n-1}$ are independent of $\left(\mathbf{x}_{n-1}, \theta\right)$ and $\mathbf{y}_{0: n-1}$ (see smoothing step above). We note here that only the (marginal) analysis pdf of $\mathbf{x}_{n}, p\left(\mathbf{x}_{n} \mid \mathbf{y}_{0: n}\right)$, is of interest since the analysis pdf of $\theta$ has already been computed in the smoothing step.

From Eq. (24), $p\left(\mathbf{x}_{n} \mid \mathbf{x}_{n-1}, \theta, \mathbf{y}_{0: n}\right)=p\left(\mathbf{x}_{n} \mid \mathbf{x}_{n-1}, \theta, \mathbf{y}_{n}\right)$. Thereby, there is a similarity between Eq. (23) and the forecast step (Eq. 17) in the sense that Eq. (23) can be seen as a forecast step once the observation $\mathbf{y}_{n}$ is known; i.e., Eq. (23) coincides with "Eq. (17) given the observation $\mathbf{y}_{n}$ ". Accordingly, and without abuse of language, we refer to Eqs. (23)-(24) as the forecast step.

\subsection{Ensemble formulation}

Since it is not possible to derive the analytical solution of Eqs. (21)-(24) because of the nonlinear character of the model, $\mathcal{M}($.$) , we use Properties 1$ and 2 (see Appendix A) to propose an EnKF-like formulation, assuming that $p\left(\mathbf{y}_{n}\right.$, $\left.\mathbf{z}_{n-1} \mid \mathbf{y}_{0: n-1}\right)$ is Gaussian for all $n$. This assumption implies that $p\left(\mathbf{z}_{n-1} \mid \mathbf{y}_{0: n-1}\right), p\left(\mathbf{z}_{n-1} \mid \mathbf{y}_{0: n}\right)$, and $p\left(\mathbf{y}_{n} \mid \mathbf{y}_{0: n-1}\right)$ are Gaussian.

\subsubsection{Smoothing step}

Starting at time $t_{n-1}$, from an analysis ensemble, $\left\{\mathbf{x}_{n-1}^{\mathrm{a},(m)}\right.$, $\left.\theta_{\left.\right|_{n-1}}^{(m)}\right\}_{m=1}^{N_{\mathrm{e}}}$, one can use Property 1 in Eq. (22) to sample the observation forecast ensemble, $\left\{\mathbf{y}_{n}^{\mathrm{f},(m)}\right\}_{m=1}^{N_{\mathrm{e}}}$, as

$\mathbf{x}_{n}^{\mathrm{f},(m)}=\mathcal{M}_{n-1}\left(\mathbf{x}_{n-1}^{\mathrm{a},(m)}, \theta_{\mid n-1}^{(m)}\right)+\eta_{n-1}^{(m)}$,

$\mathbf{y}_{n}^{\mathrm{f},(m)}=\mathbf{H}_{n} \mathbf{x}_{n}^{\mathrm{f},(m)}+\varepsilon_{n}^{(m)}$,

with $\eta_{n-1}^{(m)} \sim \mathcal{N}\left(\mathbf{0}, \mathbf{Q}_{n-1}\right)$ and $\varepsilon_{n}^{(m)} \sim \mathcal{N}\left(\mathbf{0}, \mathbf{R}_{n}\right)$. Property 2 is then used in Eq. (21) to compute the smoothing ensemble, $\left\{\mathbf{x}_{n-1}^{\mathrm{s},(m)}, \theta_{\left.\right|_{n}}^{(m)}\right\}_{m=1}^{N_{\mathrm{e}}}$, as

$\mathbf{x}_{n-1}^{\mathrm{s},(m)}=\mathbf{x}_{n-1}^{\mathrm{a},(m)}+\mathbf{P}_{x_{n-1}^{\mathrm{a}}, y_{n}^{\mathrm{f}}} \underbrace{\mathbf{P}_{y_{n}^{\mathrm{f}}}^{-1}\left(\mathbf{y}_{n}-\mathbf{y}_{n}^{\mathrm{f},(m)}\right)}_{v_{n}^{(m)}}$,

$\theta_{\mid n}^{(m)}=\theta_{\mid n-1}^{(m)}+\mathbf{P}_{\theta_{\mid n-1}, y_{n}^{\mathrm{f}}} \cdot v_{n}^{(m)}$.

The (cross-) covariances in Eqs. (27) and (28) are defined and evaluated similarly to Eqs. (9)-(11).

\subsubsection{Forecast step}

The analysis ensemble, $\left\{\mathbf{x}_{n}^{\mathrm{a},(m)}\right\}_{m=1}^{N_{\mathrm{e}}}$, can be obtained from $\left\{\mathbf{x}_{n-1}^{\mathrm{s},(m)}, \theta_{\left.\right|_{n}}^{(m)}\right\}_{m=1}^{N_{\mathrm{e}}}$ using Property 1 in Eq. (23), once the a posteriori transition pdf, $p\left(\mathbf{x}_{n} \mid \mathbf{x}_{n-1}, \theta, \mathbf{y}_{n}\right)$, is computed via Eq. (24). Furthermore, one can verify that Eq. (24) leads to a Gaussian pdf:

$$
\begin{array}{r}
p\left(\mathbf{x}_{n} \mid \mathbf{x}_{n-1}, \theta, \mathbf{y}_{n}\right)=\mathcal{N}_{x_{n}}\left(\mathcal{M}_{n-1}\left(\mathbf{x}_{n-1}, \theta\right)\right. \\
\left.\quad+\widetilde{\mathbf{K}}_{n}\left(\mathbf{y}_{n}-\mathbf{H}_{n} \mathcal{M}_{n-1}\left(\mathbf{x}_{n-1}, \theta\right)\right), \widetilde{\mathbf{Q}}_{n-1}\right),
\end{array}
$$

with $\quad \widetilde{\mathbf{K}}_{n}=\mathbf{Q}_{n-1} \mathbf{H}_{n}^{T}\left[\mathbf{H}_{n} \mathbf{Q}_{n-1} \mathbf{H}_{n}^{T}+\mathbf{R}_{n}\right]^{-1} \quad$ and $\widetilde{\mathbf{Q}}_{n-1}=\mathbf{Q}_{n-1}-\widetilde{\mathbf{K}}_{n} \mathbf{H}_{n} \mathbf{Q}_{n-1}$. However, when the state dimension, $N_{x}$, is very large, the computational cost of $\widetilde{\mathbf{K}}_{n}$ and $\widetilde{\mathbf{Q}}_{n-1}$ (which may be a non-diagonal matrix even when $\mathbf{Q}_{n-1}$ is diagonal) may become prohibitive. One way to avoid this problem is to directly sample from $p\left(\mathbf{x}_{n} \mid \mathbf{x}_{n-1}, \theta\right.$, $\mathbf{y}_{n}$ ) without explicitly computing this pdf in Eq. (29). Let $\left\{\widetilde{\mathbf{x}}_{n}^{(m)}\left(\mathbf{x}_{n-1}, \theta\right)\right\}_{m=1}^{N_{\mathrm{e}}}$ denotes an ensemble of samples drawn from $p\left(\mathbf{x}_{n} \mid \mathbf{x}_{n-1}, \theta, \mathbf{y}_{n}\right)$. The notation $\widetilde{\mathbf{x}}_{n}^{(m)}\left(\mathbf{x}_{n-1}, \theta\right)$ refers to a function $\widetilde{\mathbf{x}}_{n}^{(m)}$ of $\left(\mathbf{x}_{n-1}, \theta\right)$; similar notations hold for $\widetilde{\xi}_{n}^{(m)}$ (.) and $\widetilde{\mathbf{y}}_{n}^{(m)}($.$) in Eqs. (30) and (31), respectively. Using$ Properties 1 and 2, an explicit form of such samples can be obtained as (see Appendix B)

$$
\begin{aligned}
\widetilde{\xi}_{n}^{(m)}\left(\mathbf{x}_{n-1}, \theta\right) & =\mathcal{M}_{n-1}\left(\mathbf{x}_{n-1}, \theta\right)+\eta_{n-1}^{(m)} ; \eta_{n-1}^{(m)} \\
& \sim \mathcal{N}\left(\mathbf{0}, \mathbf{Q}_{n-1}\right), \\
\widetilde{\mathbf{y}}_{n}^{(m)}\left(\mathbf{x}_{n-1}, \theta\right) & =\mathbf{H}_{n} \widetilde{\xi}_{n}^{(m)}\left(\mathbf{x}_{n-1}, \theta\right)+\varepsilon_{n}^{(m)} ; \varepsilon_{n}^{(m)} \\
& \sim \mathcal{N}\left(\mathbf{0}, \mathbf{R}_{n}\right),
\end{aligned}
$$

$$
\begin{aligned}
\widetilde{\mathbf{x}}_{n}^{(m)}\left(\mathbf{x}_{n-1}, \theta\right)= & \widetilde{\xi}_{n}^{(m)}\left(\mathbf{x}_{n-1}, \theta\right)+\mathbf{P}_{\widetilde{\xi}_{n}, \widetilde{y}_{n}} \mathbf{P}_{\widetilde{y}_{n}}^{-1} \\
& {\left[\mathbf{y}_{n}-\widetilde{\mathbf{y}}_{n}^{(m)}\left(\mathbf{x}_{n-1}, \theta\right)\right], }
\end{aligned}
$$

where the (cross)-covariances, $\mathbf{P}_{\widetilde{\xi}_{n}, \widetilde{y}_{n}}$ and $\mathbf{P}_{\widetilde{y}_{n}}$, are evaluated from the ensembles $\left\{\widetilde{\xi}_{n}^{(m)}\left(\mathbf{x}_{n-1}, \theta\right)\right\}_{m=1}^{N_{\mathrm{e}}}$ and $\left\{\widetilde{\mathbf{y}}_{n}^{(m)}\left(\mathbf{x}_{n-1}\right.\right.$, $\theta)\}_{m=1}^{N_{\mathrm{e}}}$, similarly to Eqs. (9)-(11). Now, using Property 1 in Eq. (23), one can compute an analysis ensemble, $\left\{\mathbf{x}_{n}^{\mathrm{a},(m)}\right\}_{m=1}^{N_{\mathrm{e}}}$, from the smoothing ensemble, $\left\{\mathbf{x}_{n-1}^{\mathrm{s},(m)}, \theta_{\left.\right|_{n}}^{(m)}\right\}_{m=1}^{N_{\mathrm{e}}}$, using the functional form Eq. (32). More precisely, we obtain, $\mathbf{x}_{n}^{\mathrm{a},(m)}=\widetilde{\mathbf{x}}_{n}^{(m)}\left(\mathbf{x}_{n-1}^{\mathrm{s},(m)}, \theta_{\mid n}^{(m)}\right)$, which is equivalent to set $\mathbf{x}_{n-1}=\mathbf{x}_{n-1}^{\mathrm{s},(m)}$ and $\theta=\theta_{\mid n}^{(m)}$ in Eqs. (30)-(32). 


\subsubsection{Summary of the dual EnKF OSA $_{\text {algorithm }}$}

Starting from an analysis ensemble, $\left\{\mathbf{x}_{n-1}^{\mathrm{a},(m)}, \theta_{\mid n-1}^{(m)}\right\}_{m=1}^{N_{\mathrm{e}}}$, at time $t_{n-1}$, the updated ensemble of both state and parameters at time $t_{n}$ is obtained with the following two steps:

- Smoothing step: the state forecast ensemble, $\left\{\mathbf{x}_{n}^{\mathrm{f},(m)}\right\}_{m=1}^{N_{\mathrm{e}}}$, is first computed by Eq. (25), and then used to compute the observation forecast ensemble, $\left\{\mathbf{y}_{n}^{\mathrm{f},(m)}\right\}_{m=1}^{N_{\mathrm{e}}}$, as in Eq. (26). The observation forecast ensemble is then used to compute the one-step-ahead smoothing ensemble of the state, $\left\{\mathbf{x}_{n-1}^{\mathrm{s},(m)}\right\}_{m=1}^{N_{\mathrm{e}}}$, and parameters, $\left\{\theta_{l}^{(m)}\right\}_{m=1}^{N_{\mathrm{e}}}$, based on the Kalman-like updates (Eqs. 27 and 28), respectively.

- Forecast step: the analysis ensemble of the state $\left\{\mathbf{x}_{n}^{\mathrm{a},(m)}\right\}_{m=1}^{N_{\mathrm{e}}}$ is obtained as

$$
\begin{aligned}
\xi_{n}^{(m)} & =\mathcal{M}_{n-1}\left(\mathbf{x}_{n-1}^{\mathrm{s},(m)}, \theta_{\mid n}^{(m)}\right)+\eta_{n-1}^{(m)} ; \eta_{n-1}^{(m)} \\
& \sim \mathcal{N}\left(\mathbf{0}, \mathbf{Q}_{n-1}\right), \\
\widetilde{\mathbf{y}}_{n}^{\mathrm{f},(m)} & =\mathbf{H}_{n} \xi_{n}^{(m)}+\varepsilon_{n}^{(m)} ; \varepsilon_{n}^{(m)} \sim \mathcal{N}\left(\mathbf{0}, \mathbf{R}_{n}\right), \\
\mathbf{x}_{n}^{\mathrm{a},(m)} & =\xi_{n}^{(m)}+\mathbf{P}_{\xi_{n}, \widetilde{y}_{n}^{\mathrm{f}}} \mathbf{P}_{\widetilde{y}_{n}^{f}}^{-1}\left(\mathbf{y}_{n}-\widetilde{\mathbf{y}}_{n}^{\mathrm{f},(m)}\right),
\end{aligned}
$$

with $\mathbf{P}_{\xi_{n}, \widetilde{y}_{n}^{\mathrm{f}}}=\left(N_{\mathrm{e}}-1\right)^{-1} \mathbf{S}_{\xi_{n}} \mathbf{S}_{\widetilde{y}_{n}^{\mathrm{f}}}^{T}$ and $\mathbf{P}_{\widetilde{y}_{n}^{\mathrm{f}}}=\left(N_{\mathrm{e}}-1\right)^{-1}$ $\mathbf{S}_{\widetilde{y}_{n}^{\mathrm{f}}} \mathbf{S}_{\tilde{y}_{n}^{\mathrm{f}}}^{T}$.

The proposed dual EnKF $\mathrm{OSA}_{\mathrm{S}}$ is an ensemble implementation, under the common Gaussian assumption, of the generic Bayesian filtering algorithm presented in Sect. 3.1. This justifies its Bayesian consistency in contrast with the standard dual EnKF, which, as discussed in Sect. 2.2.3, lacks a Bayesian interpretation. In contrast with the dual EnKF, which uses $\theta_{\mid n}^{(m)}$ and $\mathbf{x}_{n-1}^{\mathrm{a},(m)}$ to compute $\mathbf{x}_{n}^{\mathrm{a},(m)}$ (see Eq. 14), the proposed dual EnKF OSA uses $\theta_{\mid n}^{(m)}$ and the smoothed state members, $\mathbf{x}_{n-1}^{\mathrm{s},(m)}$, which are the $\mathbf{x}_{n-1}^{\mathrm{a},(m)}$ after an update with the current observation, $\mathbf{y}_{n}$, following Eq. (27). Therefore, when including the Kalman-like correction term as well, the observation, $\mathbf{y}_{n}$, is used 3 times in the dual $\mathrm{EnKF}_{\mathrm{OSA}}$ in a fully consistent Bayesian formulation, compared to only twice in the dual EnKF. This means that the dual EnKF ${ }_{\text {OSA }}$ exploits the observations more efficiently than the dual EnKF, which should provide more information for improved and more consistent state and parameters estimates. Note that the dual EnKF $_{\mathrm{OSA}}$ reduces to the dual EnKF in the particular case of a perfect model and $\mathbf{x}_{n-1}^{\mathrm{s},(m)}=\mathbf{x}_{n-1}^{\mathrm{a},(m)}$.

The joint EnKF OSA $_{\text {of }}$ Gharamti et al. (2015) has been derived following the same approach under the assumption of independence between the state, $\mathbf{x}_{n}$, and its observation, $\mathbf{y}_{n}$, given the previous state, $\mathbf{x}_{n-1}$, and parameters, $\theta$ (assumption (16) in Gharamti et al., 2015). This assumption has been adopted to avoid evaluating the computationally demanding term $p\left(\mathbf{x}_{n} \mid \mathbf{x}_{n-1}, \theta, \mathbf{y}_{n}\right)$ by replacing it with the more easily sampled state transition pdf, $p\left(\mathbf{x}_{n} \mid \mathbf{x}_{n-1}\right.$, $\theta)=\mathcal{N}_{x_{n}}\left(\mathcal{M}_{n-1}\left(\mathbf{x}_{n-1}, \theta\right), \mathbf{Q}_{n-1}\right)$, to draw the state analysis ensemble. Here, we propose a more efficient approach to directly sample the analysis ensemble without explicitly computing $p\left(\mathbf{x}_{n} \mid \mathbf{x}_{n-1}, \theta, \mathbf{y}_{n}\right)$ and without the need of any additional assumption. The joint EnKF $\mathrm{OSA}_{\mathrm{SA}}$ is therefore a particular case of the dual EnKF $F_{O S A}$, involving two Kalman-like updates only (those of the smoothing step), since in the forecast step, the state analysis members, $\mathbf{x}_{n}^{\mathrm{a},(m)}$, are computed from the smoothed members, $\left(\mathbf{x}_{n-1}^{\mathrm{s},(m)}, \theta_{\mid n}^{(m)}\right)$, by integrating them with the model and without any update with the current observation. More specifically, Eqs. (33)-(35) above reduce in Gharamti et al. (2015) to Eq. (33) (i.e., $\mathbf{x}_{n}^{\mathrm{a},(m)}=\xi_{n}^{(m)}$ ).

Despite the smoothing formulation of the dual EnKF $F_{O S A}$, this algorithm obviously addresses the state forecast problem as well. As discussed in the smoothing step above, the (onestep-ahead) forecast members are inherently computed. The $j$-step-ahead forecast member, denoted by $\mathbf{x}_{n+j \mid n}^{(m)}$ for $j \geq 2$, can be computed following a recursive procedure where, for $\ell=2,3, \cdots, j$, one has

$$
\begin{aligned}
\mathbf{x}_{n+\ell \mid n}^{(m)} & =\mathcal{M}_{n+\ell-1}\left(\mathbf{x}_{n+\ell-1 \mid n}^{(m)}, \theta_{\mid n}^{(m)}\right)+\eta_{n+\ell-1}^{(m)}, \eta_{n+\ell-1}^{(m)} \\
& \sim \mathcal{N}\left(\mathbf{0}, \mathbf{Q}_{n+\ell-1}\right) .
\end{aligned}
$$

\subsection{Complexity of the joint EnKF, dual EnKF, and dual EnKF OSA}

The computational complexity of the different stateparameter EnKF schemes can be split between the forecast (time-update) step and the analysis (measurement-update) step. The joint EnKF requires $N_{\mathrm{e}}$ model runs (for forecasting the state ensemble) and $N_{\mathrm{e}}$ Kalman corrections (for updating the forecast ensemble). This is practically doubled when using the dual EnKF, since the latter requires $2 N_{\mathrm{e}}$ model runs and $2 N_{\mathrm{e}}$ Kalman corrections: $N_{\mathrm{e}}$ corrections for each of the forecast state ensemble and the forecast parameter ensemble. As presented in the previous section, the dual EnKF ${ }_{\mathrm{OSA}}$ smoothes the state estimate at the previous time step before updating the parameters and the state at the current time. Thus, the dual EnKF $F_{O S A}$ requires as many model runs $\left(2 N_{\mathrm{e}}\right)$ as the dual EnKF, and an additional $N_{\mathrm{e}}$ correction steps to apply smoothing. In large-scale geophysical applications, the correction step of the ensemble members is often computationally not significant compared to the cost of integrating the model in the forecast step. The approximate computational complexity and memory storage for each algorithm are summarized in Table 1. The tabulated complexities for each method are valid under the assumption that $N_{y} \ll N_{x}$; i.e., the number of state variables is much larger than the number of observations. This is generally the case for subsurface flow applications due to budget constraints given the consequent costs needed for drilling and maintaining subsurface wells. 
Table 1. Approximate computational complexities of the joint EnKF, the dual EnKF, and the dual EnKF OSA algorithms. Notations are as follows. $N_{x}$ : number of state variables, $N_{\theta}$ : number of parameter variables, $N_{y}$ : number of observations, $N$ : number of assimilation cycles, $N_{\mathrm{e}}$ : ensemble size, $\mathcal{C}_{x}$ : state model cost (i.e., $N_{x}^{2}$ is the linear $\mathrm{KF}$ ), $\mathcal{C}_{\theta}$ : parameter model cost (usually free $\equiv$ identity), $\mathcal{C}_{y}$ : observation operator cost (i.e., $N_{y} N_{x}$ in the linear $\mathrm{KF}$ ), $\mathcal{S}_{x}$ : storage volume for one state vector, and $\mathcal{S}_{\theta}$ : storage volume for one parameter vector.

\begin{tabular}{llll}
\hline Algorithm & Time update & Measurement update & Storage \\
\hline joint EnKF & $N N_{\mathrm{e}}\left(\mathcal{C}_{x}+\mathcal{C}_{\theta}\right)$ & $N N_{\mathrm{e}}\left(\mathcal{C}_{y}+N_{y} N_{\theta}\right)+N N_{\mathrm{e}}^{2}\left(N_{x}+N_{\theta}\right)$ & $2 N N_{\mathrm{e}}\left(\mathcal{S}_{x}+\mathcal{S}_{\theta}\right)$ \\
dual EnKF & $N N_{\mathrm{e}}\left(2 \mathcal{C}_{x}+\mathcal{C}_{\theta}\right)$ & $2 N N_{\mathrm{e}} \mathcal{C}_{y}+N N_{\mathrm{e}}^{2}\left(N_{x}+N_{\theta}\right)$ & $2 N N_{\mathrm{e}}\left(\mathcal{S}_{x}+\mathcal{S}_{\theta}\right)$ \\
dual EnKF $\mathrm{OSA}$ & $N N_{\mathrm{e}}\left(2 \mathcal{C}_{x}+\mathcal{C}_{\theta}\right)$ & $2 N N_{\mathrm{e}} \mathcal{C}_{y}+N N_{\mathrm{e}}^{2}\left(2 N_{x}+N_{\theta}\right)$ & $2 N N_{\mathrm{e}}\left(\mathcal{S}_{x}+\mathcal{S}_{\theta}\right)$ \\
\hline
\end{tabular}

\section{Numerical experiments}

\subsection{Transient groundwater flow problem}

We adopt in this study the subsurface flow problem of Bailey and Baú (2010). The system consists of a 2-D transient flow with an areal aquifer area of $0.5 \mathrm{~km}^{2}$ (Fig. 1). Constant head boundaries of 20 and $15 \mathrm{~m}$ are placed on the west and east ends of the aquifer, respectively, with an average saturated thickness, $b$, of $25 \mathrm{~m}$. The height of the impermeable aquifer bottom, $z_{\text {bot }}$, is assumed constant (i.e., horizontal aquifer bottom). The north and south boundaries are assumed to be impermeable (Fig. 1). The mesh is discretized using a cell-centered finite difference scheme with $10 \mathrm{~m} \times 20 \mathrm{~m}$ rectangles, resulting in 2500 elements. The following 2-D saturated groundwater flow system is solved:

$$
\frac{\partial}{\partial x}\left(T_{x} \frac{\partial h}{\partial x}\right)+\frac{\partial}{\partial y}\left(T_{y} \frac{\partial h}{\partial y}\right)=S \frac{\partial h}{\partial t}+q,
$$

where $T$ is the transmissivity $\left[\mathrm{L}^{2} \mathrm{~T}^{-1}\right]$, which is related to the conductivity, $K$, through $T=K b, h$ is the hydraulic head [L], $t$ is time [T], $S$ is storativity [-], and $q$ denotes the sources as recharge or sinks due to pumping wells $\left[\mathrm{L} \mathrm{T}^{-1}\right]$. Unconfined aquifer conditions are simulated by setting $S=0.20$ to represent the specific yield. A logconductivity field is generated using the sequential Gaussian simulation toolbox, GCOSIM3D (Gómez-Hernández and Journel, 1993), with a geometric mean of $10^{-13} \mathrm{~m} \mathrm{~s}^{-1}$, a variance of $Y=\log K$ equal to 1.5 , and a Gaussian variogram with a range equal to $250 \mathrm{~m}$ in the $x$ direction and $500 \mathrm{~m}$ in the $y$ direction (Fig. 1).

We consider a dynamically complex experimental setting involving various time-dependent external forcings. The recharge is assumed spatially heterogenous and sampled using the GCOSIM3D toolbox (Gómez-Hernández and Journel, 1993) with statistical parameters shown in Table 2. Three different pumping wells (PW) are inserted within the aquifer domain and can be seen in Fig. 1 (square symbols). From these wells, transient pumping of groundwater takes place with different daily values as plotted in the left panel of Fig. 2. The highest pumping rates are associated with PW2 with an average daily rate of $0.0513 \mathrm{~m} \mathrm{day}^{-1}$. Smaller

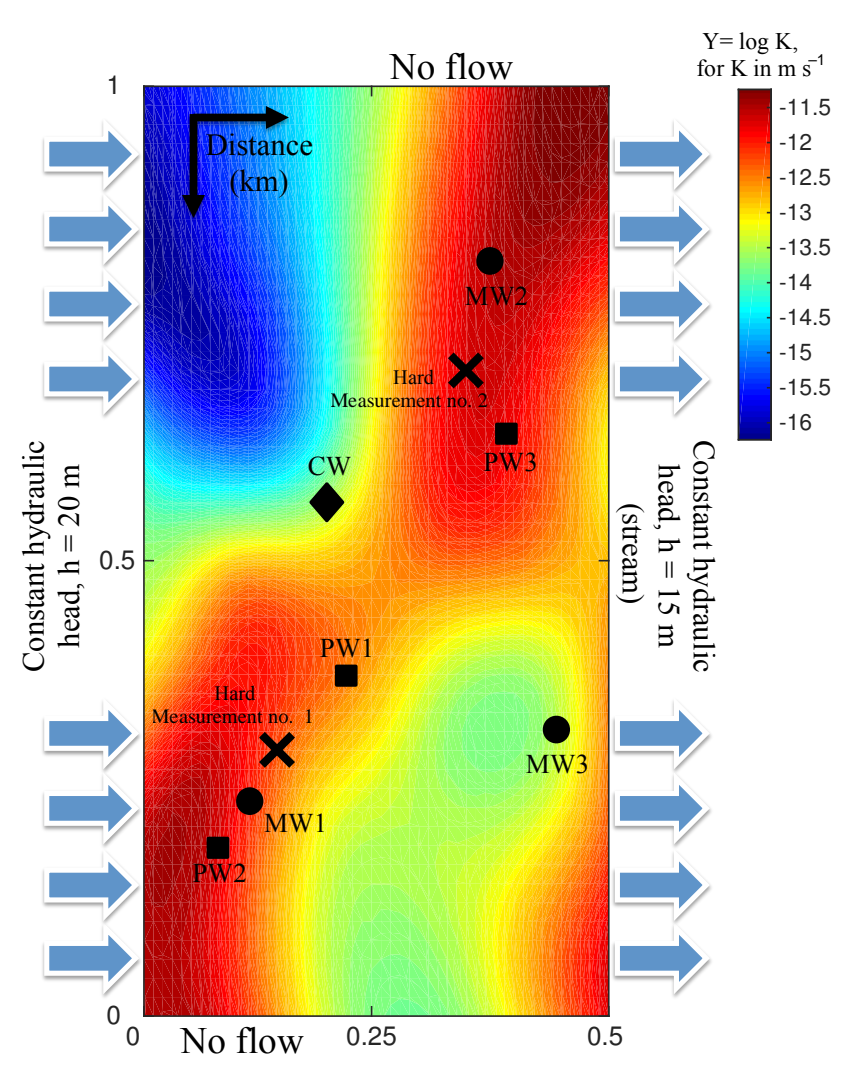

Figure 1. Plan view of the conceptual model for the 2-D transient groundwater flow problem. East and west boundaries ( $x$ direction) are Dirichlet with a given prescribed hydraulic heads. North and south boundaries ( $y$ direction) are impermeable (no flow boundaries). The reference log-conductivity field was obtained using the sequential Gaussian simulation code (Gómez-Hernández and Journel, 1993). A Gaussian variogram model is considered with a geometric mean of $10^{-13} \mathrm{~m} \mathrm{~s}^{-1}$, a variance of $Y=\log K$ equal to 1.5 , and range equal to 250 and $500 \mathrm{~m}$ in the $x$ and $y$ directions, respectively. The black squares represent the pumping wells, whereas the black circles denote the position of three monitoring wells. The black diamond is a control well. The two black crosses correspond to the locations where the conductivity values were used to condition the geostatistical simulation. 
Table 2. Parameters of the random functions for modeling the spatial distributions of the reference and perturbed recharge fields. The ranges in $x$ and $y$ directions for the variogram model are given by $\lambda_{x}$ and $\lambda_{y}$, respectively. $\tau$ denotes the rotation angle of one clockwise rotation around the positive $y$ axis.

\begin{tabular}{lcclcrc}
\hline Recharge & Mean & Variance & Variogram & $\lambda_{x}$ & $\lambda_{y}$ & $\tau$ \\
\hline Reference field & $-20 \mathrm{~m} \mathrm{~s}^{-1}$ & 1.03 & Gaussian & $50 \mathrm{~m}$ & $100 \mathrm{~m}$ & $45^{\circ}$ \\
Perturbed field & $-20 \mathrm{~m} \mathrm{~s}^{-1}$ & 1.21 & Gaussian & $50 \mathrm{~m}$ & $50 \mathrm{~m}$ & $45^{\circ}$ \\
\hline
\end{tabular}
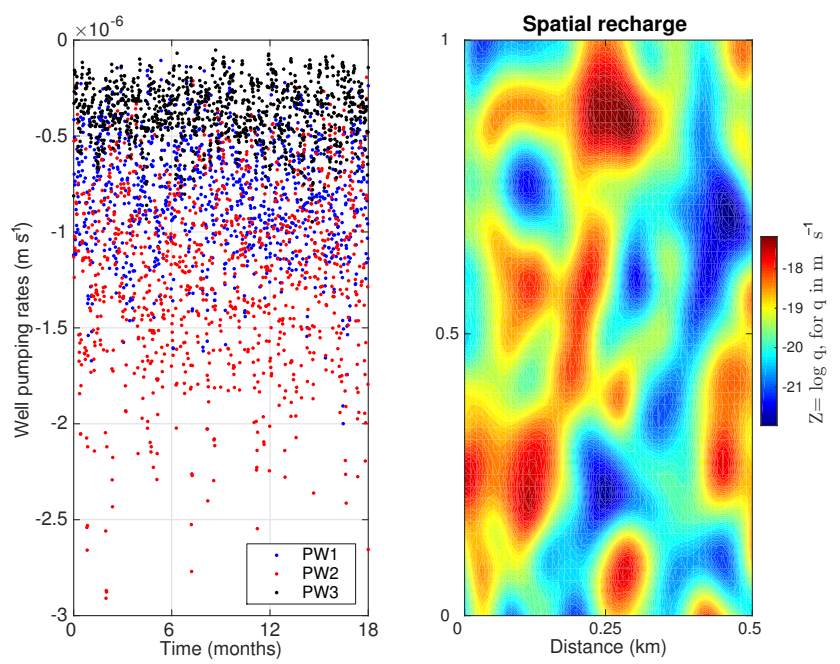

Figure 2. Left panel: daily transient reference pumping rates from wells PW1, PW2, and PW3. Negative values indicate pumping or groundwater that is being removed from the aquifer. Right panel: reference heterogenous spatial recharge values obtained using the sequential Gaussian simulation code (Gómez-Hernández and Journel, 1993) with parameters given in Table 2.

temporal variations in water pumping rates are assigned to PW1 and PW3. Three other monitoring wells (MW1, MW2, MW3) are also placed within the aquifer domain to evaluate the groundwater flow filters estimates. We further assess the prediction skill of the model after data assimilation using a control well $(\mathrm{CW})$ placed in the middle of the aquifer (indicated by a diamond symbol). The assigned values for the hydraulic conductivity and recharge rates might be smaller than what is generally used in real-world applications. This, however, should not affect the performance of the tested schemes.

Prior to assimilation, a reference run is first conducted for each experimental setup using the prescribed parameters above, and is considered as the truth. We simulate the groundwater flow system over a year-and-a-half period using the classical fourth-order Runge-Kutta method with a time step of $12 \mathrm{~h}$. The initial hydraulic head configuration is obtained after a 2-years model spin-up starting from a uniform $15 \mathrm{~m}$ head. Reference heterogenous recharge rates are used in the setup as explained before. The water head changes (in $\mathrm{m}$ ) after 18 months are displayed with contour lines in the left panel of Fig. 3. One can notice larger variations in the wa-
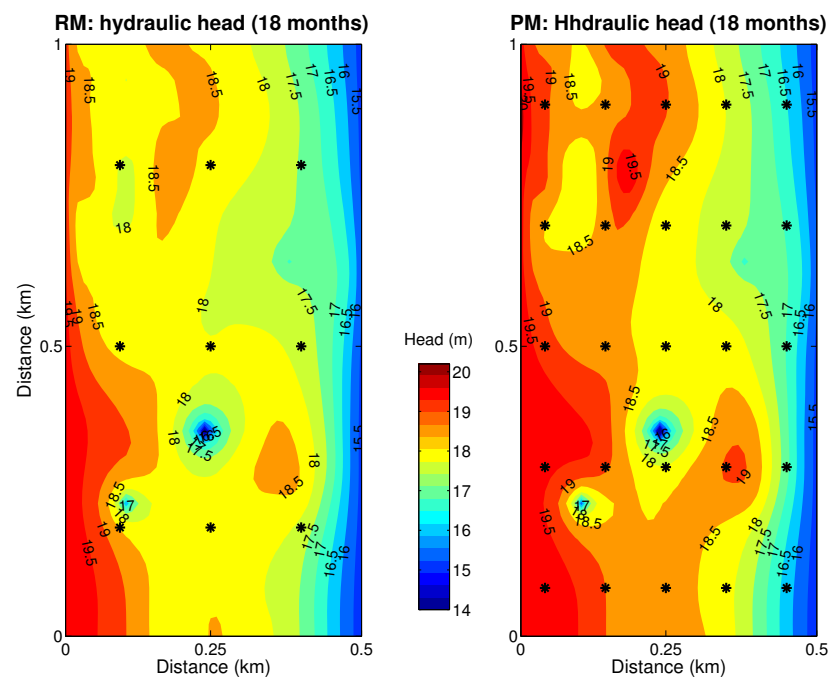

Figure 3. Groundwater flow contour maps obtained using the reference run (left panel) and the perturbed forecast model (right panel) after 18 months of simulation. The well locations from which head data are extracted are shown by black asterisks. In the left panel, we show the first network consisting of nine wells. In the right panel, the other network with 25 wells is displayed.

ter head in the lower left corner of the aquifer domain, consistent with the high conductivity values in that region. The effects of transient pumping in addition to the heterogenous recharge rates are also well observed in the vicinity of the pumping wells.

\subsection{Assimilation experiments}

To imitate a realistic setting, we impose various perturbations on the reference model and set our goal to estimate the water head and the hydraulic conductivity fields using an imperfect forecast model and perturbed data extracted from the reference (true) run. This experimental framework is known as "twin-experiments". In the forecast model, we perturb both transient pumping and spatial recharge rates. The perturbed recharge field, as compared to the reference recharge in Fig. 2, is sampled with different variogram parameters as shown in Table 2. Pumping rates from PW1, PW2, and PW3 are perturbed by adding a Gaussian noise with mean zero and standard deviation equal to $20 \%$ of the reference transient rates. The flow field simulated by the forecast (perturbed) 
model after 18 months is shown in the right panel of Fig. 3. Compared to the reference field, there are clear spatial differences in the hydraulic head, especially around the first and second pumping wells.

To demonstrate the effectiveness of the proposed dual $\mathrm{EnKF}_{\mathrm{OSA}}$, we evaluate its performances against the standard joint and dual EnKFs under different experimental scenarios. We further conduct a number of sensitivity experiments, changing (1) the ensemble size, (2) the temporal frequency of available observations, (3) the number of observation wells in the domain, and (4) the measurement error. For the frequency of the observations, we consider six scenarios in which hydraulic head measurements are extracted from the reference run every $1,3,5,10,15$, and 30 days. Of course, $\mathbf{x}_{n}^{\mathrm{a},(m)}$ is equal to $\mathbf{x}_{n}^{\mathrm{f},(m)}$ when no observation is assimilated. We also test four different observational networks assuming 9, 15,25 , and 81 wells uniformly distributed throughout the aquifer domain (Fig. 3 displays two of these networks; with 9 and 25 wells). We evaluate the algorithms under 10 different scenarios in which the observations were perturbed with Gaussian noise of zero mean and a standard deviation equal to $0.05,0.10,0.15,0.20,0.25,0.30,0.50,1,2$, and $3 \mathrm{~m}$. Such measurement errors, which can be due to instruments errors, conversion of pressure to water head, or piezometer well defects, are typical values (order of centimeters to meters) observed at real hydrologic sites (Post and von Asmuth, 2013).

To initialize the filters, we follow Gharamti et al. (2014a) and perform a 5-year (spin-up) run using the perturbed forecast model starting from the mean hydraulic head, $\bar{h}_{\mathrm{REF}}$, of the reference run solution. $\bar{h}_{\mathrm{REF}}$ is calculated as the temporal mean at every grid cell of the reference run snapshots (a total of 1095 , retained every $12 \mathrm{~h}$ ). After 5 years, a set of 3650 head maps are obtained. From these, we randomly select $N_{\mathrm{e}}$ head maps and use it as the initial hydraulic head ensemble. By doing so, the dynamic head changes that may occur in the aquifer are well represented by the initial ensemble. The corresponding parameters' realizations are sampled with the geostatistical software, GCOSIM3D, using the same variogram parameters of the reference conductivity field but conditioned on two hard measurements as indicated by black crosses in Fig. 1. The two data points capture some parts of the high conductivity regions in the domain, and thus one should expect a poor representation of the low conductivity areas in the initial $\log (K)$ ensemble. This is a challenging case for the filters especially when a sparse observational network is considered. To ensure consistency between the hydraulic heads and the conductivities at the beginning of the assimilation, we conduct a spin-up of the whole stateparameters ensemble for a 6-months period using perturbed recharge time series for each ensemble member.

The filter estimates resulting from the different filters are evaluated based on their average absolute forecast er- rors (AAE) and their average ensemble spread (AESP):

$$
\begin{gathered}
\mathrm{AAE}=N_{z}^{-1} N_{\mathrm{e}}^{-1} \sum_{j=1}^{N_{\mathrm{e}}} \sum_{i=1}^{N_{z}}\left|\mathbf{z}_{j, i}^{\mathrm{f}, \mathrm{e}}-\mathbf{z}_{i}^{\mathrm{t}}\right|, \\
\mathrm{AESP}=N_{z}^{-1} N_{\mathrm{e}}^{-1} \sum_{j=1}^{N_{\mathrm{e}}} \sum_{i=1}^{N_{z}}\left|\mathbf{z}_{j, i}^{\mathrm{f}, \mathrm{e}}-\hat{\mathbf{z}}_{i}^{\mathrm{f}, \mathrm{e}}\right|,
\end{gathered}
$$

where $\mathbf{z}_{i}^{\mathrm{t}}$ is the reference "true" value of the variable (state or parameter) at cell $i, \mathbf{z}_{j, i}^{\mathrm{f}, \mathrm{e}}$ is the forecast ensemble value of the variable, and $\hat{\mathbf{z}}_{i}^{\mathrm{f}, \mathrm{e}}$ is the forecast ensemble mean at location $i$. AAE measures the estimate-truth misfit and AESP measures the ensemble spread, or the confidence in the estimated values (Hendricks Franssen and Kinzelbach, 2008). $N_{z}$ is the total number of variables in the domain and equal to $N_{x}$ or $N_{\theta}$. We further assess the accuracy of the estimates by plotting the resulting field and variance maps of both hydraulic head and conductivities.

\section{Results and discussion}

\subsection{Sensitivity to the ensemble size}

We first study the sensitivity of the three algorithms to the ensemble size, $N_{\mathrm{e}}$. In realistic groundwater applications, we would be restricted to small ensembles due to computational limitations. Obtaining accurate state and parameter estimations with small ensembles is thus desirable. We carry the experiments using three ensemble sizes, $N_{\mathrm{e}}=50,100$, and 300, and we fix the period of the observations to half a day, the number of wells to nine (Fig. 3, left observation network), and the measurement error standard deviation to $0.50 \mathrm{~m}$. We plot the resulting AAE time series of the state and parameters in Fig. 4. As shown, the performance of the joint EnKF, dual EnKF, joint EnKF $F_{O S A}$, and dual EnKF $F_{O S A}$ improves as the ensemble size increases, reaching a mean AAE of $0.161,0.160$, and $0.156 \mathrm{~m}$ for $N_{\mathrm{e}}=300$, respectively. The joint EnKF and the dual EnKF exhibit similar behaviors, with a slight advantage for the dual EnKF. As argued by Gharamti et al. (2014a), the dual EnKF is generally expected to produce more accurate results only when large enough ensembles are used. We have tested the joint and the dual EnKFs using 1000 members and found that the dual EnKF is around 9\% more accurate in terms of AAE. The proposed dual EnKF $O$ AsA provides the best estimates in all tested scenarios. The joint EnKF ${ }_{O S A}$ outperforms the joint and dual EnKFs, but is about $5 \%$ less accurate than the dual $\mathrm{EnKF}_{\mathrm{OSA}}$, especially after the first year of assimilation. On average, with changing ensemble size, the dual EnKF $\mathrm{OSA}_{\mathrm{A}}$ leads to about $7 \%$ improvement compared with the standard joint and dual schemes. In terms of the conductivity estimates, the proposed scheme produces more accurate estimates for all three ensemble sizes. At the early assimilation stage, the four schemes seem to provide similar results, but 
Table 3. Mean average ensemble spread (AESP) of the water head and the hydraulic conductivity for three different ensemble sizes. The reported values are given for the joint EnKF, dual EnKF, joint EnKF $F_{O S A}$, and the proposed dual EnKF ${ }_{O S A}$.

\begin{tabular}{lcccccccc}
\hline & \multicolumn{3}{c}{ Hydraulic head } & & \multicolumn{3}{c}{ Conductivity } \\
\cline { 2 - 3 } \cline { 7 - 9 } & $N_{\mathrm{e}}=50$ & $N_{\mathrm{e}}=100$ & $N_{\mathrm{e}}=300$ & & $N_{\mathrm{e}}=50$ & $N_{\mathrm{e}}=100$ & $N_{\mathrm{e}}=300$ \\
\hline Joint EnKF & 0.123 & 0.144 & 0.200 & & 1.076 & 1.014 & 0.951 \\
Dual EnKF & 0.126 & 0.145 & 0.201 & & 1.075 & 1.016 & 0.951 \\
Joint EnKF & 0.125 & 0.145 & 0.201 & & 1.026 & 0.977 & 0.908 \\
Dual EnKF & 0.117 & 0.141 & 0.183 & & 1.039 & 0.907 & 0.879 \\
\hline
\end{tabular}

Table 4. Filter inbreeding indicator: Ratio of the mean average absolute error (AAE) and mean average ensemble spread (AESP) of the water head and the hydraulic conductivity for three different ensemble sizes. The reported values are given for the joint EnKF, dual EnKF, and the proposed dual EnKF $F_{\text {OSA }}$.

\begin{tabular}{lccccccc}
\hline & \multicolumn{3}{c}{ Hydraulic head } & & \multicolumn{3}{c}{ Conductivity } \\
\cline { 2 - 4 } \cline { 6 - 8 } & $N_{\mathrm{e}}=50$ & $N_{\mathrm{e}}=100$ & $N_{\mathrm{e}}=300$ & & $N_{\mathrm{e}}=50$ & $N_{\mathrm{e}}=100$ & $N_{\mathrm{e}}=300$ \\
\hline Joint EnKF & 1.734 & 1.680 & 1.619 & & 1.539 & 1.507 & 1.134 \\
Dual EnKF & 1.449 & 1.443 & 1.360 & & 1.123 & 1.123 & 0.834 \\
Dual EnKF & 0.805 & 0.802 & 0.854 & & 0.793 & 0.792 & 0.801 \\
\hline
\end{tabular}

this eventually changes after 6 months beyond which the dual EnKF $_{\text {OSA }}$ clearly outperforms the other schemes.

Furthermore, we examined the estimated uncertainties about the forecast estimates by computing the average spread of both the hydraulic head and conductivity ensembles. To do this, we evaluated the time-averaged AESP of both variables and tabulated the results for the three ensemble sizes in Table 3. For all schemes, increasing the ensemble would increase the spread of the hydraulic head ensemble due to the natural variability of the considered subsurface system. In contrast, the AESP conductivity decreases as $N_{\mathrm{e}}$ increases, probably because of the persistence nature of its prescribed dynamics. The dual EnKF ${ }_{\mathrm{OSA}}$ has the smallest mean AESP for all cases, suggesting more confidence in the head and conductivity estimates.

One could also exploit the computed AAE and AESP to assess whether the filters suffer from the inbreeding problem. Filter inbreeding occurs when the variance of the state and parameters ensemble is increasingly reduced over time. This may not only deteriorate the quality of the estimated filter error covariance matrices, but also wrongly suggests more confidence in the forecast and strongly limits the filter update by the incoming observation. One standard test for examining inbreeding is to compute the ratio of the AAE to the AESP (Hendricks Franssen and Kinzelbach, 2008). In a well designed assimilation system (that does not suffer from inbreeding) such a ratio should be close to one; in other words, the AAE and AESP are almost of the same order. Examining Fig. 4 and Table 4, the ratio of the AAE to the AESP for the different tested ensemble sizes is, on-average, very close to 1 for all three schemes, as reported in Table 4. This clearly suggests that no filtering inbreeding issues are encountered in
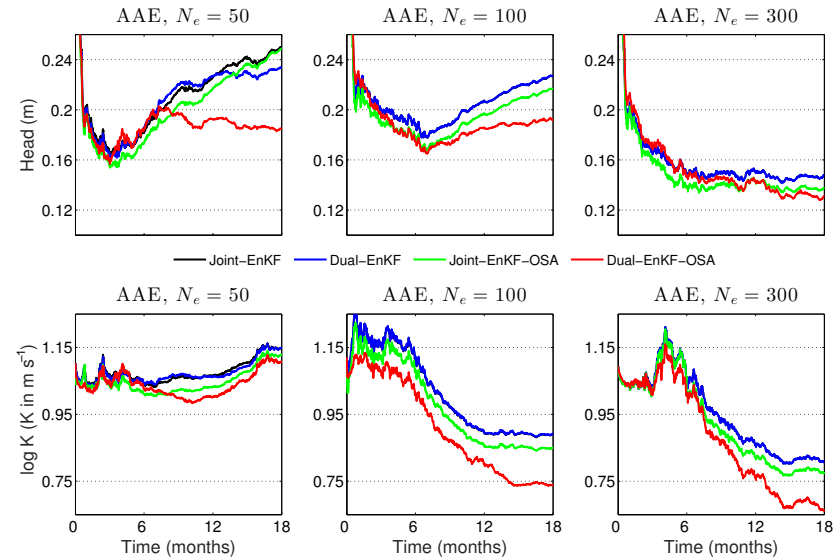

Figure 4. AAE time series of the hydraulic head and conductivity using the joint EnKF, dual EnKF, joint EnKF $F_{\text {OSA }}$, and dual

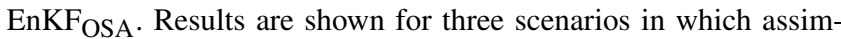
ilation of hydraulic head data are obtained from nine wells every 0.5 days. The three experimental scenarios use 50,100, and 300 ensemble members with $0.50 \mathrm{~m}$ as the measurement error standard deviation.

the present setup. This could be due to the imposed stochastic model errors (as described in Sect. 4), which seems to maintain enough spread in the hydraulic head and conductivity ensembles. Another method for tackling the inbreeding problem is to combine the EnKF with the so-called stochastic moments equations that govern the time evolution of conditional expectations of the state and parameters as well as the associated covariances, as suggested by Panzeri et al. (2013, 2015). 
In terms of computational cost, we note that our assimilation results were obtained using a $2.30 \mathrm{GHz}$ workstation and four cores for parallel looping while integrating the ensemble members. The joint EnKF is the least intensive requiring $70.61 \mathrm{~s}$ to perform a year-and-a-half assimilation run using 50 members. The dual EnKF and dual EnKF OSA, on the other hand, require 75.37 and $77.04 \mathrm{~s}$, respectively. The dual EnKF is computationally more demanding than the joint EnKF because it includes an additional propagation step of the ensemble members as discussed in Sect. 3.3. Likewise, the proposed dual EnKF $\mathrm{OSA}_{\mathrm{S}}$ requires both an additional propagation step and an update step of the state members. Its computational complexity is thus greater than the joint scheme and roughly equivalent to that of the dual EnKF. Note that in the current setup the cost of integrating the groundwater model is not very significant as compared to the cost of the update step. This is due to the simplified structure of the utilized hydrological model. This, however, should not hold for largescale hydrological applications.

\subsection{Sensitivity to the frequency of observations}

In the second set of experiments, we test the filters' behavior with different temporal frequency of observations; i.e., the times at which head observations are assimilated. We implement the three filters with 100 members and use data from nine observation wells perturbed with $0.10 \mathrm{~m}$ noise.

Figure 5 plots the mean AAE of the hydraulic conductivity estimated using the three filters for the six different observations sampling frequencies. The dual and joint EnKFs lead to comparable performances, but the latter performs slightly better when data are assimilated more frequently, i.e., every 5 and 3 days. The performance of the proposed dual EnKF $F_{O S A}$, as seen from the plot, is rather good and its estimates are more consistent with the data than those computed by the other two filters. The best dual EnKF OSA results are obtained when assimilating data every 1,3 , and 5 days. The improvements over the joint and the dual schemes decrease as observations are sampled less frequently in time. The reason for this is related to the nature of the dual EnKF $F_{O S A}$ algorithm, which adds a one-step-ahead smoothing to the analyzed head ensemble members before updating the forecast parameters and the state samples. Therefore, the more data are available, the greater the number of applied smoothing steps, and hence the better the characterization of the state and parameters. To illustrate, the smoothing step of the state ensemble enhances its statistics and eventually provides more consistent state-parameters cross-correlations to better predict the data. When assimilating hydraulic head data on a daily basis, the proposed dual $\mathrm{EnKF}_{\mathrm{OSA}}$ leads to about $24 \%$ more accurate conductivity estimates than the joint and dual EnKFs.

We have also compared the hydraulic head estimates for different sampling frequencies of observations. Similar to the parameters, the improvements of the dual EnKF $_{\mathrm{OSA}}$ algorithm over the other schemes become significant when more

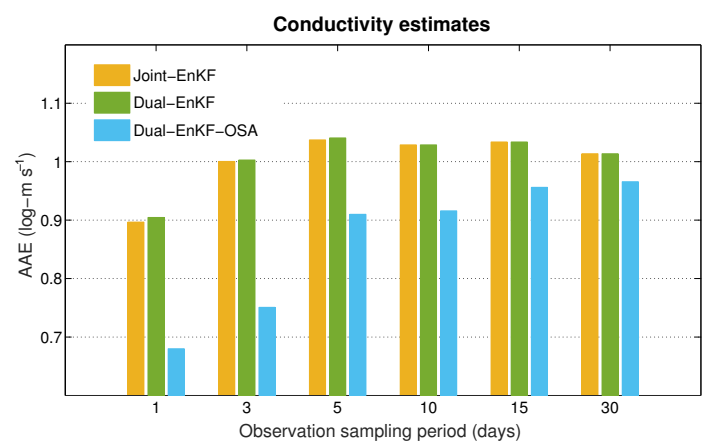

Figure 5. Mean average absolute errors (AAE) of log-hydraulic conductivity, $\log (K)$, obtained using the joint EnKF, dual EnKF, and dual EnKF $F_{O S A}$ schemes. Results are shown for six different scenarios in which assimilation of hydraulic head data are obtained from nine wells every $1,3,5,10,15$, and 30 days. All six experimental scenarios use 100 ensemble members and $0.10 \mathrm{~m}$ as the measurement error standard deviation.

data are assimilated over time. Overall, the benefits of the proposed scheme seem to be more pronounced for the estimation of the parameters, probably because the conductivity values at all aquifer cells are indirectly updated using hydraulic head data, requiring more observations for efficient estimation.

One effective way to evaluate the estimates of the state is to examine the evolution of the reference heads and the forecast ensemble members at various aquifer locations. For this, we plot in Fig. 6 the true and the estimated time-series change in hydraulic head at the assigned monitoring wells as they result from the joint EnKF, dual EnKF, and the dual EnKF $_{\mathrm{OSA}}$. We use 100 ensemble members and assume the nine data points are available every 5 days. At MW1, the performance of the three filters is quite similar and they all successfully reduce the uncertainties and recover the true evolution of the hydraulic head at that location. We note that between the 5th and the 9th month, the dual EnKF seems to underestimate the reference values of the hydraulic head as compared to the other two schemes. At MW2 and MW3, the ensemble spread of all three filters shrinks shortly after the start of assimilation, but remains larger than those at MW1. The proposed dual EnKF $\mathrm{FSA}_{\mathrm{O} \text { a }}$ well recovers the reference trajectory at MW2 and MW3. The ensemble head values obtained using the joint and the dual EnKFs at MW2 are less accurate. Furthermore, the joint and the dual EnKF ensemble members tend to underestimate the reference hydraulic head at MW3 over the first 6 months of assimilation. Beyond this, there is a clear overestimation of the head values, especially by the dual EnKF, up to the end of the first year.

\subsection{Sensitivity to the number of observations}

We further examine the robustness of the proposed dual EnKF $_{\mathrm{OSA}}$ against the joint and dual EnKFs to different num- 

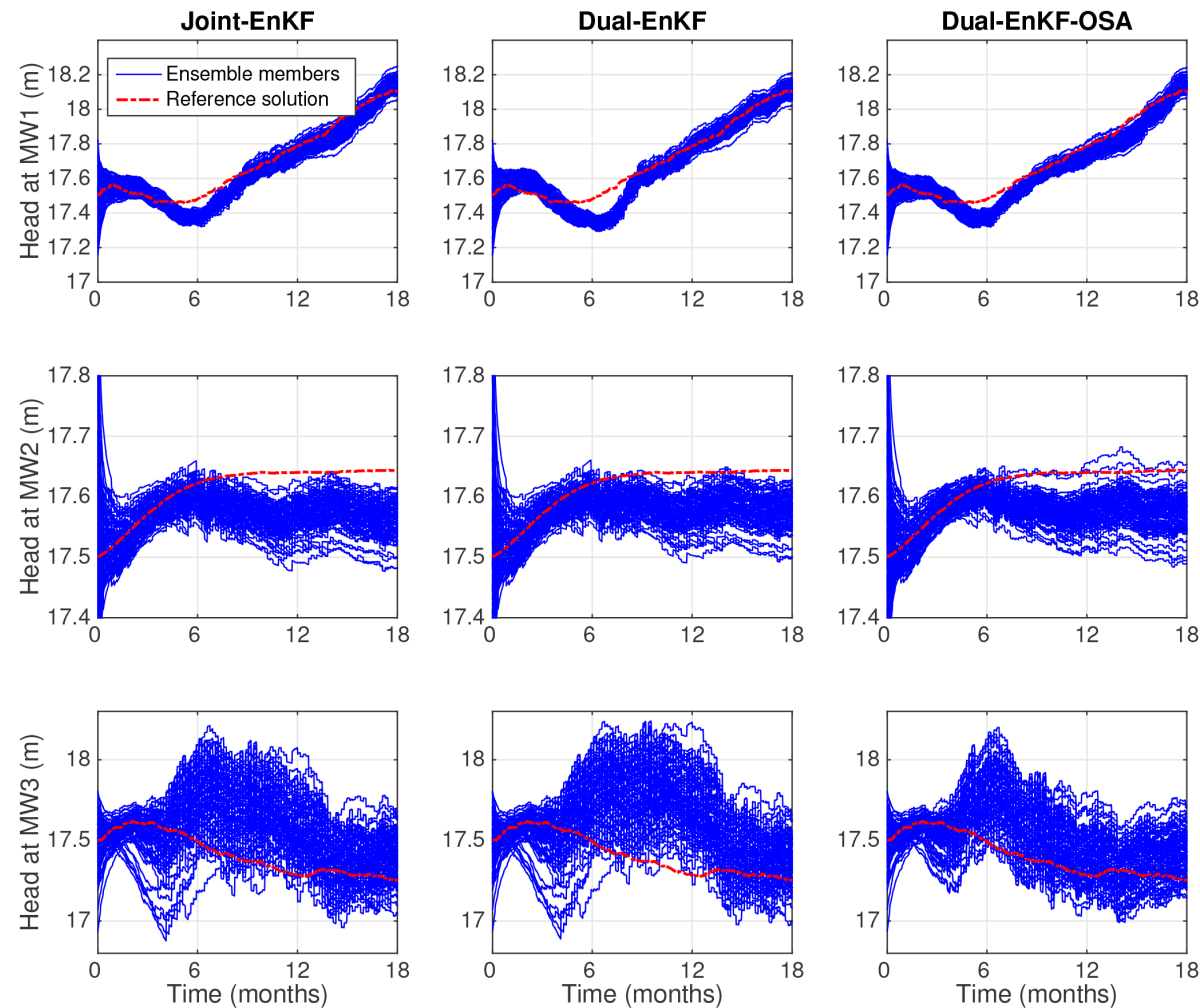

Figure 6. Reference (dashed) and predicted (solid) hydraulic head evolution at monitoring wells MW1, MW2, and MW3. Results are obtained using the joint EnKF and the dual EnKF OSA schemes with 100 members, 5 days as sampling period, nine observation wells, and $0.10 \mathrm{~m}$ of measurement noise.
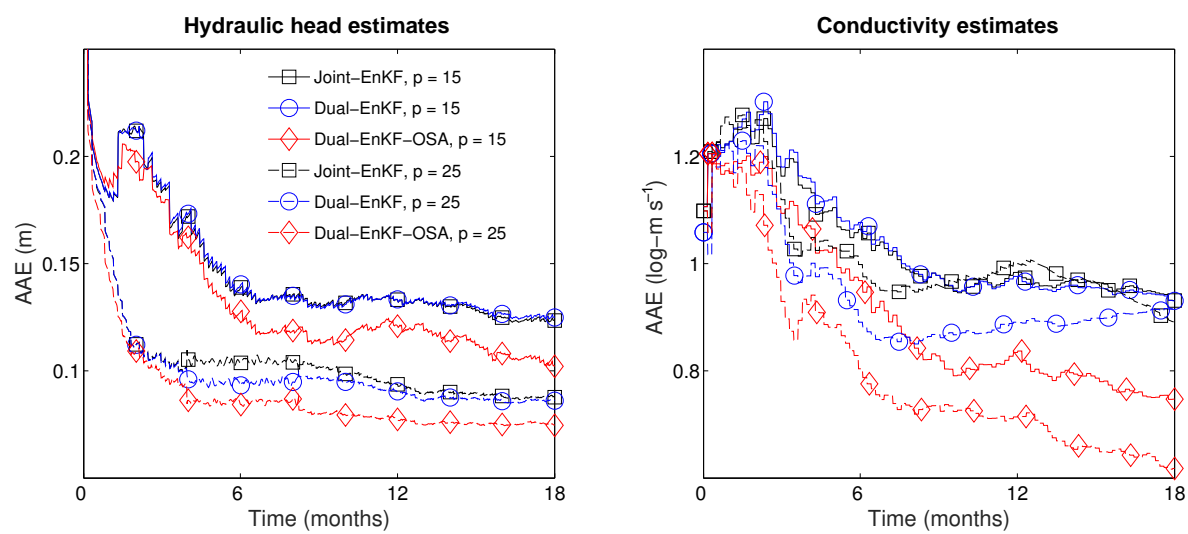

Figure 7. Time series of AAE of hydraulic head (left panel) and conductivity (right panel) using the joint EnKF, dual EnKF, and dual EnKF $_{\text {OSA }}$ schemes. Results are shown for two scenarios in which assimilation of hydraulic head data are obtained from 15 and 25 wells (uniformly distributed throughout the aquifer domain) every 5 days. The four experimental scenarios use 100 ensemble members and $0.10 \mathrm{~m}$ as the measurement error standard deviation. The number of wells is denoted by $p$.

bers of observation wells inside the aquifer domain. We thus compare our earlier estimates resulting from only nine wells, 5 days sampling period, and $0.10 \mathrm{~m}$ measurement error standard deviation with a new set of estimates resulting from more dense observational networks with 15,25 , and 81 wells. Figure 7 plots the time-series curves of the AAE as they re- sult from the four observational scenarios for hydraulic head and conductivity. As shown, the behavior of the filters improves as more data are assimilated. Clearly, the proposed scheme provides the best estimates over the entire simulation window. More precisely, and towards the end of assimilation, the dual EnKF $\mathrm{OSA}_{\mathrm{S}}$ with only 9 data points exhibits fewer 

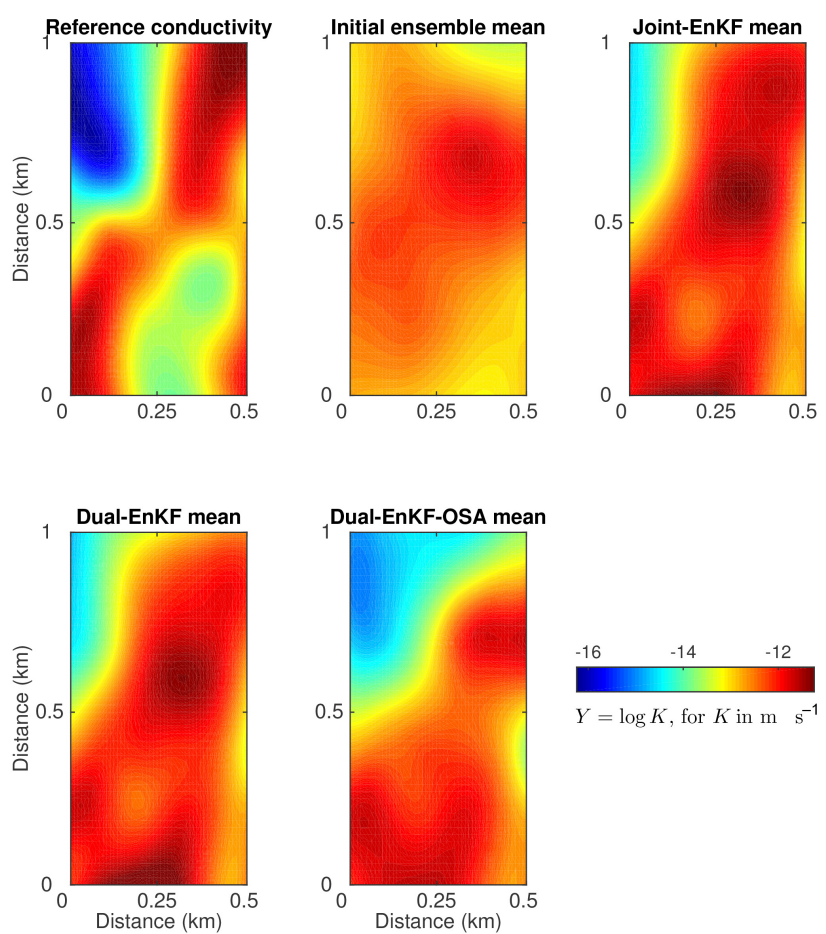

Figure 8. Spatial maps of the reference, initial and recovered ensemble means of hydraulic conductivity using the joint EnKF, dual

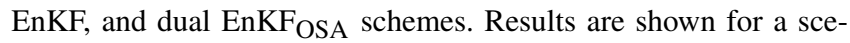
nario in which assimilation of hydraulic head data is obtained from nine wells every 5 days. This experiment uses 100 ensemble members and $0.10 \mathrm{~m}$ as the measurement error standard deviation.

forecast errors for conductivity than does the dual EnKF (and joint EnKF) with 81 data points. Likewise when assimilating head data from 15 and 25 wells, the proposed algorithm outperforms the joint and dual EnKFs and yields more accurate hydraulic head estimates by the end of the simulation window.

To further assess the performance of the filters we analyze the spatial patterns of the estimated fields. To do so, we plot and interpret the ensemble mean of the conductivity as it results from the three filters using nine observation wells. We compare the estimated fields after 18 months (Fig. 8) with the reference conductivity. As can be seen, the joint and the dual EnKFs exhibit some overshooting in the southern (low conductivity) and central regions of the domain. In contrast, the dual EnKF $\mathrm{OSA}_{\mathrm{A}}$ better delineates these regions and further provides reasonable estimates of the low conductivity area in the northwest part of the aquifer. In general and for all tested schemes, the estimated conductivity field does not capture very well the spatial variability of the reference field. This is due to the large model errors imposed on the recharge and pumping rates during the forecasts. This limits the efficiency of the assimilation system, especially with the recovery of small-scale conductivity structures, but also allows for more straightforward assessment of the different techniques.

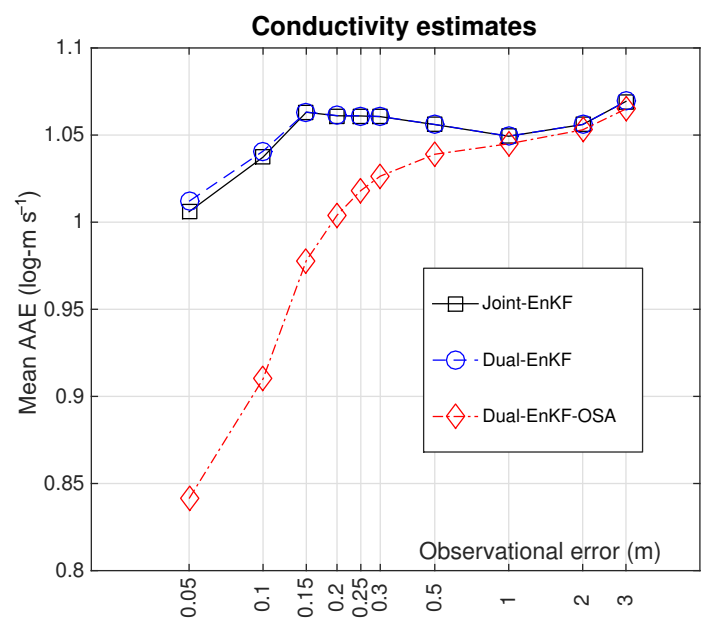

Figure 9. Mean AAE of the hydraulic conductivity using the joint EnKF, dual EnKF, and dual EnKF OSA $_{\text {s }}$ schemes. Results are shown for 10 different scenarios in which assimilation of hydraulic head data is performed using nine wells with measurement error standard deviations of $0.05,0.10,0.15,0.20,0.25,0.3,0.5,1,2$, and $3 \mathrm{~m}$. The four experimental scenarios use 100 ensemble members and 5 days as sampling period. The $x$ axis is displayed in log scale.

\subsection{Sensitivity to measurement errors}

In the last set of sensitivity experiments, we fix the number of wells to nine, the sampling period to 5 days, and test with different standard deviations of measurement error to perturb the observations. We plot the results of 10 different observational error scenarios in Fig. 9 and compare the conductivity estimates obtained using the joint EnKF, dual EnKF, and the dual EnKF $F_{O S A}$. In general, the performance of the filters appears to degrade as the observations are perturbed with a larger degree of noise. All three filters exhibit similar performances with large observational error; i.e., 1, 2, and $3 \mathrm{~m}$. This can be expected because larger observational errors decrease the impact of data assimilation, and may reduce the estimation process to a model prediction only. The plot also suggests that the estimates of the dual EnKF OSA with $0.30 \mathrm{~m}$ measurement error standard deviation are better than those of the joint and the dual EnKFs with $0.10 \mathrm{~m}$ error. With $0.10 \mathrm{~m}$ measurement error standard deviation, the estimate of the dual EnKF $\mathrm{OSA}_{\mathrm{SA}}$ is also approximately $12 \%$ better.

Finally, we investigated the time evolution of the ensemble variance of the conductivity estimates as they result from the dual EnKF and the dual EnKF $F_{O S A}$ with $0.10 \mathrm{~m}$ measurement noise. Spatially, the ensemble variance maps provide insight about the uncertainty reduction due to data assimilation. The initial map (Fig. 10, left panel) exhibits zero variance at the sampled two locations and increasing variance away from these locations. The ensemble spread of conductivity field from the two filters (Fig. 10, right panels) after 6 and 18 months is quite small and comparable. The dual EnKF $_{\mathrm{OSA}}$, however, tends to maintain a larger variance to- 

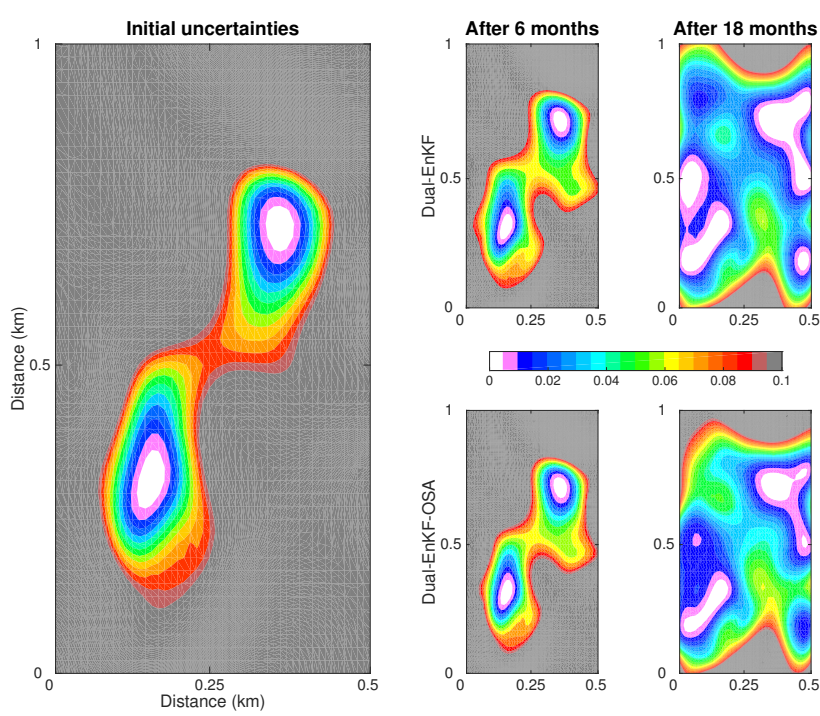

Figure 10. Left panel: ensemble variance map of the initial conductivity field. Right sub-panels: ensemble variance maps of estimated conductivity after 6 and 18 month assimilation periods using the dual EnKF and the proposed dual EnKFOSA schemes. These results are obtained with 100 members, 5 days of sampling period, nine observation wells, and $0.10 \mathrm{~m}$ as measurement noise.

wards the north edges than the dual EnKF, which in turn helps increase the weight of the observations in this area.

\subsection{Prediction capability assessment}

To further assess the system performance in terms of parameters retrieval, we have integrated the model in prediction mode (without assimilation) for an additional period of 18 months starting from the end of the assimilation period. We plot in Fig. 11, using the final estimates of the conductivity as they result from the three filters (after 18 months), the time evolution of the hydraulic head at the CW. The ensemble size is set to 100 , sampling period is 1 day, number of data wells is 25 , and measurement noise is $0.5 \mathrm{~m}$. The reference head trajectory at the $\mathrm{CW}$ decreases from 17.5 to $16.9 \mathrm{~m}$ in the first 2 years, and then slightly increases to $17.2 \mathrm{~m}$ in the rest of the years. The forecast ensemble members of the joint EnKF at this CW fail to capture to reference trajectory of the model. This is due to the large measurement noise imposed on the head data. The dual EnKF performs slightly better and predicts hydraulic head values that are closer to the reference solution. The performance of the dual EnKF $\mathrm{KSA}_{\text {A }}$, as shown, is the closest to the reference head trajectory and, moreover, one of the forecast ensemble members successfully captures the true head evolution. We further plot the absolute bias of the hydraulic head during the prediction phase, i.e., after 1.5 years, using the three filtering schemes. As shown, the bias in the joint EnKF reaches about $0.6 \mathrm{~m}$ after 3 years. On the other hand, the dual EnKF $\mathrm{FSA}_{\mathrm{SA}}$ and, to a lesser extent, the dual EnKF, clearly lead to more accurate long-term forecasts
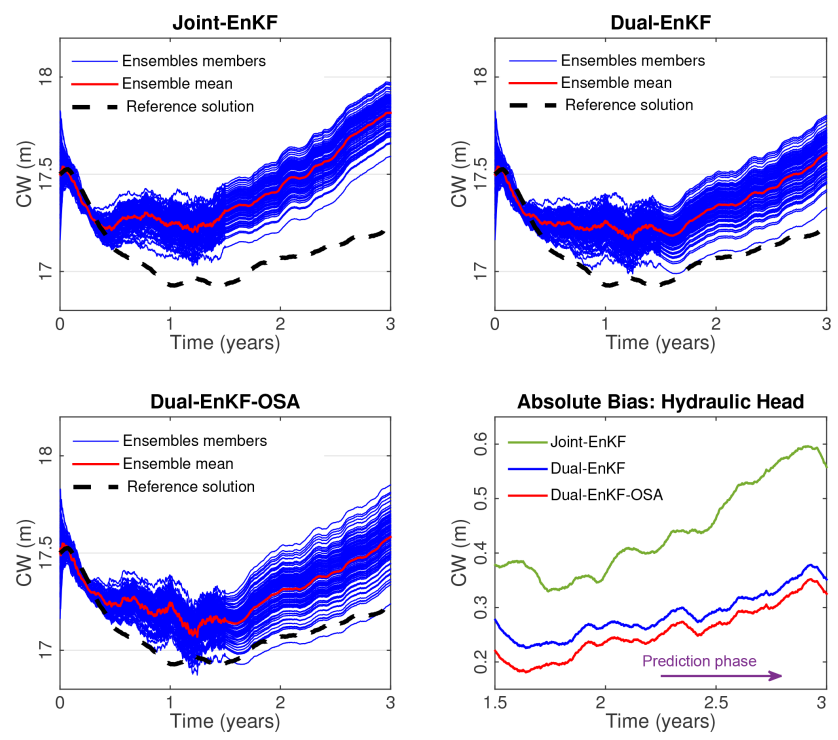

Figure 11. Reference (dashed) and predicted (solid) hydraulic head evolution at the control well: CW. Results are obtained using the

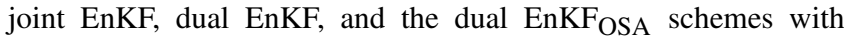
100 members, 1 day as sampling period, 25 observation wells, and $0.50 \mathrm{~m}$ of measurement noise. The last 18 months are purely based on the forecast model prediction with no assimilation of data. In the bottom-right subplot, the absolute bias of hydraulic head is evaluated for all schemes during the prediction phase only (i.e., after 1.5 years).

with smaller bias in the resulting hydraulic head estimates. A similar tests was also conducted at other locations in the aquifer, all resulting in similar conclusions.

Finally, in order to demonstrate that our results are statistically robust, 10 other test cases with different reference conductivity and heterogeneous recharge maps were investigated. In each of these cases, we sampled the reference fields by varying the variogram parameters, such as variance, $x$ and $y$ ranges, etc. The pumping rates and the initial head configuration among the cases were also altered. For all 10 test cases, we fixed the ensemble size to 100 and used data from nine observation wells every 3 days. We set the measurement error standard deviation to $0.10 \mathrm{~m}$. We plot the resulting conductivity estimates (mean AAE) from each case in Fig. 12. As shown in the plot, the estimates of the three filters give a statistical evidence that the proposed scheme always provides more accurate estimates than the joint/dual EnKF and is more robust to changing dynamics and experimental setups. Similar results were obtained for the hydraulic head estimates. Averaging over all test cases, the proposed scheme provides about $17 \%$ more accurate estimates in term of AAE than the standard joint and dual EnKFs. 


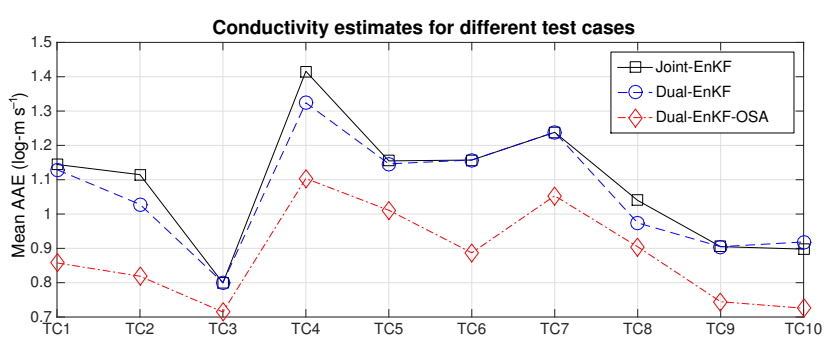

Figure 12. Performance of the joint/dual EnKF and the proposed dual EnKF $F_{\text {OSA }}$ schemes in 10 different test cases (TC1, TC2, etc.). Mean AAE of the conductivity estimates are displayed. These results are obtained with 100 members, 3 days of sampling period, nine observation wells, and $0.10 \mathrm{~m}$ as measurement noise.

\section{Conclusions}

We presented a one-step-ahead smoothing-based dual ensemble Kalman filter (dual EnKF ${ }_{O S A}$ ) for state-parameter estimation of subsurface groundwater flow models. The dual EnKF $_{O S A}$ is derived using a Bayesian probabilistic formulation combined with two classical stochastic sampling properties. It differs from the standard joint EnKF and dual EnKF in the fact that the order of the time-update step of the state (forecast by the model) and the measurement-update step (correction by the incoming observations) is inverted. Compared with the dual EnKF, this introduces a smoothing step to the state by future observations, which seems to provide the model, at the time of forecasting, with better and rather physically consistent state and parameters ensembles.

We tested the proposed dual EnKFOSA on a conceptual groundwater flow model in which we estimated the hydraulic head and spatially variable conductivity parameters. We conducted a number of sensitivity experiments to evaluate the accuracy and the robustness of the proposed scheme and to compare its performance against those of the standard joint and dual EnKFs. The experimental results suggest that the dual EnKF $\mathrm{OSA}_{\mathrm{S}}$ is more robust, successfully estimating the hydraulic head and the conductivity field under different modeling scenarios. Sensitivity analyses demonstrate that when more observations are assimilated, the dual EnKF $\mathrm{KSA}_{\mathrm{O}}$ becomes more effective and significantly outperforms the standard joint and dual EnKF schemes. In addition, when using a sparse observation network in the aquifer domain, the accuracy of the dual EnKF OSA estimates is better preserved, unlike the dual EnKF, which seems to be more sensitive to the number of hydraulic wells. Moreover, the dual EnKF $_{O S A}$ results are shown to be more robust against observation noise. On average, the dual EnKF $\mathrm{KSA}_{\mathrm{SA}}$ scheme leads to around $10 \%$ more accurate state and parameter solutions than those resulting from the standard joint and dual EnKFs.

The proposed scheme is easy to implement and only requires minimal modifications to a standard EnKF code. It is further computationally feasible, requiring only a marginal increase in the computational cost compared to the dual
EnKF. This scheme should therefore be beneficial to the hydrology community given its consistency, high accuracy, and robustness to changing modeling conditions. It could serve as an efficient estimation tool for real-world problems, such as groundwater, contaminant transport, and reservoir monitoring, in which the available data are often sparse and noisy. Potential future research includes testing the dual EnKF $O$ OSA with realistic large-scale groundwater, contaminant transport and reservoir monitoring problems. Furthermore, combining the proposed state-parameter estimation scheme with other iterative and hybrid ensemble approaches may be a promising direction for further improvements.

\section{Data availability}

Only simulated data were used in this study. Please contact the corresponding or first author for the details about the algorithms and the codes. 


\section{Appendix A: Some useful properties of random sampling}

The following classical results of random sampling are extensively used in the derivation of the ensemble-based filtering algorithms presented in this paper.

Property 1 (Hierarchical sampling; Robert, 2007). Assuming that one can sample from $p\left(\mathbf{x}_{1}\right)$ and $p\left(\mathbf{x}_{2} \mid \mathbf{x}_{1}\right)$, then a sample, $\mathbf{x}_{2}^{*}$, from $p\left(\mathbf{x}_{2}\right)$ can be drawn as follows:

1. $\mathbf{x}_{1}^{*} \sim p\left(\mathbf{x}_{1}\right)$

2. $\mathbf{x}_{2}^{*} \sim p\left(\mathbf{x}_{2} \mid \mathbf{x}_{1}^{*}\right)$.

Property 2 (conditional sampling; Hoffman and Ribak, 1991). Consider a Gaussian pdf, $p(\mathbf{x}, \mathbf{y})$, with $\mathbf{P}_{x y}$ and $\mathbf{P}_{y}$ denoting the cross-covariance of $\mathbf{x}$ and $\mathbf{y}$ and the covariance of $\mathbf{y}$, respectively. Then a sample, $\mathbf{x}^{*}$, from $p(\mathbf{x} \mid \mathbf{y})$, can be drawn as follows:

1. $(\widetilde{\mathbf{x}}, \widetilde{\mathbf{y}}) \sim p(\mathbf{x}, \mathbf{y})$,

2. $\mathbf{x}^{*}=\widetilde{\mathbf{x}}+\mathbf{P}_{x y} \mathbf{P}_{y}^{-1}[\mathbf{y}-\tilde{\mathbf{y}}]$.

\section{Appendix B: Sampling of the posterior transition pdf}

We show here that the samples, $\widetilde{\mathbf{x}}_{n}^{(m)}\left(\mathbf{x}_{n-1}, \theta\right)$, given in Eq. (32), are drawn from the a posteriori transition pdf, $p\left(\mathbf{x}_{n} \mid \mathbf{x}_{n-1}, \theta, \mathbf{y}_{n}\right)$. Lets start by showing how Eqs. (30)(31) are obtained. According to Eq. (15), on can show that the members, $\widetilde{\xi}_{n}^{(m)}\left(\mathbf{x}_{n-1}, \theta\right)$, given by Eq. (30), are samples from the transition pdf, $p\left(\mathbf{x}_{n} \mid \mathbf{x}_{n-1}, \theta\right)=\mathcal{N}_{x_{n}}\left(\mathcal{M}_{n-1}\left(\mathbf{x}_{n-1}\right.\right.$, $\left.\theta), \mathbf{Q}_{n-1}\right)$. Furthermore, one may use Property 1 in Eq. (22), which is recalled here,

$$
p\left(\mathbf{y}_{n} \mid \mathbf{x}_{n-1}, \theta\right)=\int \underbrace{p\left(\mathbf{y}_{n} \mid \mathbf{x}_{n}\right)}_{\mathcal{N}_{y_{n}}\left(\mathbf{H}_{n} \mathbf{x}_{n}, \mathbf{R}_{n}\right)} \underbrace{p\left(\mathbf{x}_{n} \mid \mathbf{x}_{n-1}, \theta\right)}_{\left\{\widetilde{\xi}_{n}^{(m)}\left(\mathbf{x}_{n-1}, \theta\right)\right\}_{m=1}^{N_{\mathrm{e}}}} \mathrm{d} \mathbf{x}_{n},
$$

to obtain the members, $\widetilde{\mathbf{y}}_{n}^{(m)}\left(\mathbf{x}_{n-1}, \theta\right)$, given by Eq. (31); such members are, indeed, samples from $p\left(\mathbf{y}_{n} \mid \mathbf{x}_{n-1}, \theta\right)$.

Now, using the samples $\widetilde{\xi}_{n}^{(m)}\left(\mathbf{x}_{n-1}, \theta\right)$ of $p\left(\mathbf{x}_{n} \mid \mathbf{x}_{n-1}\right.$, $\theta)=p\left(\mathbf{x}_{n} \mid \mathbf{x}_{n-1}, \theta, \mathbf{y}_{0: n-1}\right)$ and the samples $\tilde{\mathbf{y}}_{n}^{(m)}\left(\mathbf{x}_{n-1}, \theta\right)$ of $p\left(\mathbf{y}_{n} \mid \mathbf{x}_{n-1}, \theta\right)=p\left(\mathbf{y}_{n} \mid \mathbf{x}_{n-1}, \theta, \mathbf{y}_{0: n-1}\right)$, one can apply Property 2 to the joint pdf, $p\left(\mathbf{x}_{n}, \mathbf{y}_{n} \mid \mathbf{x}_{n-1}, \theta, \mathbf{y}_{0: n-1}\right)$, assuming it is Gaussian, to show that the samples $\widetilde{\mathbf{x}}_{n}^{(m)}\left(\mathbf{x}_{n-1}, \theta\right)$, given in Eq. (32), are drawn from the a posteriori transition pdf, $p\left(\mathbf{x}_{n} \mid \mathbf{x}_{n-1}, \theta, \mathbf{y}_{0: n}\right)=p\left(\mathbf{x}_{n} \mid \mathbf{x}_{n-1}, \theta, \mathbf{y}_{n}\right)$. 
Acknowledgements. Research reported in this publication was supported by King Abdullah University of Science and Technology (KAUST).

Edited by: M. Giudici

Reviewed by: three anonymous referees

\section{References}

Alcolea, A., Carrera, J., and Medina, A.: Pilot points method incorporating prior information for solving the groundwater flow inverse problem, Adv. Water Resour., 29, 1678-1689, 2006.

Bailey, R. and Baú, D.: Ensemble smoother assimilation of hydraulic head and return flow data to estimate hydraulic conductivity distribution, Water Resour. Res., 46, W12543, doi:10.1029/2010WR009147, 2010.

Chang, S.-Y., Chowhan, T., and Latif, S.: State and parameter estimation with an SIR particle filter in a three-dimensional groundwater pollutant transport model, J. Environ. Eng., 138, 11141121, 2012.

Chen, Y. and Zhang, D.: Data assimilation for transient flow in geologic formations via ensemble Kalman filter, Adv. Water Resour., 29, 1107-1122, 2006.

Crestani, E., Camporese, M., Baú, D., and Salandin, P.: Ensemble Kalman filter versus ensemble smoother for assessing hydraulic conductivity via tracer test data assimilation, Hydrol. Earth Syst. Sci., 17, 1517-1531, doi:10.5194/hess-17-1517-2013, 2013.

Desbouvries, F., Petetin, Y., and Ait-El-Fquih, B.: Direct, Prediction- and Smoothing-based Kalman and Particle Filter Algorithms, Signal Process., 91, 2064-2077, 2011.

Doucet, A., de Freitas, N., and Gordon, N. (Eds.): Sequential Monte Carlo Methods in Practice, Statistics for Engineering and Information Science, Springer Verlag, New York, 2001.

Erdal, D. and Cirpka, O. A.: Joint inference of groundwaterrecharge and hydraulic-conductivity fields from head data using the ensemble Kalman filter, Hydrol. Earth Syst. Sci., 20, 555569, doi:10.5194/hess-20-555-2016, 2016.

Feyen, L., Vrugt, J. A., Nualláin, B. Ó., van der Knijff, J., and De Roo, A.: Parameter optimisation and uncertainty assessment for large-scale streamflow simulation with the LISFLOOD model, Journal of Hydrology, 332, 276-289, 2007.

Gharamti, M. E. and Hoteit, I.: Complex step-based low-rank extended Kalman filtering for state-parameter estimation in subsurface transport models, J. Hydrol., 509, 588-600, 2014.

Gharamti, M. E., Kadoura, A., Valstar, J., Sun, S., and Hoteit, I.: Constraining a compositional flow model with flow-chemical data using an ensemble-based Kalman filter, Water Resour. Res., 50, 2444-2467, 2014a.

Gharamti, M. E., Valstar, J., and Hoteit, I.: An adaptive hybrid EnKF-OI scheme for efficient state-parameter estimation of reactive contaminant transport models, Adv. Water Resour., 71, 1-15, 2014b.

Gharamti, M. E., Hoteit, I., and Valstar, J.: Dual states estimation of a subsurface flow-transport coupled model using ensemble Kalman filtering, Adv. Water Resour., 60, 75-88, 2013.

Gharamti, M. E., Ait-El-Fquih, B., and Hoteit, I.: An iterative ensemble Kalman filter with one-step-ahead smoothing for state- parameters estimation of contaminant transport models, J. Hydrol., 527, 442-57, 2015.

Gómez-Hernández, J. J. and Journel, A. G.: Joint sequential simulation of multigaussian fields, in: Geostatistics Troia '92, Springer, the Netherlands, Kluwer Academic Publishers, 85-94, 1993.

Hendricks Franssen, H. and Kinzelbach, W.: Real-time groundwater flow modeling with the Ensemble Kalman Filter: Joint estimation of states and parameters and the filter inbreeding problem, Water Resour. Res., 44, W09408, doi:10.1029/2007WR006505, 2008.

Hendricks Franssen, H. and Kinzelbach, W.: Ensemble Kalman filtering versus sequential self-calibration for inverse modelling of dynamic groundwater flow systems, J. Hydrol., 365, 261-274, 2009.

Hoffman, Y. and Ribak, E.: Constrained realizations of Gaussian fields - a simple algorithm, Astrophys. J., 380, L5-L8, 1991.

Hoteit, I., Pham, D.-T., Triantafyllou, G., and Korres, G.: A New Approximate Solution of the Optimal Nonlinear Filter for Data Assimilation in Meteorology and Oceanography, Mon. Weather Rev., 136, 317-334, 2008.

Lee, W. and Farmer, C.: Data Assimilation by Conditioning of Driving Noise on Future Observations, IEEE T. Signal Process., 62, 3887-3896, 2014.

Li, L., Zhou, H., Gómez-Hernández, J. J., and Hendricks Franssen, H.-J.: Jointly mapping hydraulic conductivity and porosity by assimilating concentration data via ensemble Kalman filter, J. Hydrol., 428, 152-169, 2012.

Lü, H., Yua, Z., Zhu, Yonghua, D.-S., Hao, Z., and Sudicky, A. E.: Dual state-parameter estimation of root zone soil moisture by optimal parameter estimation and extended Kalman filter data assimilation, Adv. Water Resour., 34, 395-406, 2011.

Lü, H., Hou, T., Horton, R., Zhu, Y., Chen, X., Jia, Y., Wang, W., and $\mathrm{Fu}, \mathrm{X}$. : The streamflow estimation using the Xinanjiang rainfall runoff model and dual state-parameter estimation method, J. Hydrol., 480, 102-114, 2013.

McLaughlin, D.: An integrated approach to hydrologic data assimilation: interpolation, smoothing, and filtering, Adv. Water Resour., 25, 1275-1286, 2002.

McMillan, H. K., Hreinsson, E. Ö., Clark, M. P., Singh, S. K., Zammit, C., and Uddstrom, M. J.: Operational hydrological data assimilation with the recursive ensemble Kalman filter, Hydrol. Earth Syst. Sci., 17, 21-38, doi:10.5194/hess-17-21-2013, 2013.

Montzka, C., Moradkhani, H., Weihermüller, L., Franssen, H.-J. H., Canty, M., and Vereecken, H.: Hydraulic parameter estimation by remotely-sensed top soil moisture observations with the particle filter, J. Hydrol., 399, 410-421, 2011.

Moradkhani, H., Hsu, K.-L., Gupta, H., and Sorooshian, S.: Uncertainty assessment of hydrologic model states and parameters: Sequential data assimilation using the particle filter, Water Resour. Res., 41, W05012, doi:10.1029/2004WR003604, 2005a.

Moradkhani, H., Sorooshian, S., Gupta, H. V., and Houser, P. R.: Dual state - parameter estimation of hydrological models using ensemble Kalman filter, Adv. Water Resour., 28, 135-147, 2005 b.

Nævdal, G., Johnsen, L. M., Aanonsen, S. I., and Vefring, E. H.: Reservoir monitoring and continuous model updating using ensemble Kalman filter, Soc. Petrol. Eng. J., 10, 66-74, 2005.

Panzeri, M., Riva, M., Guadagnini, A., and Neuman, S. P.: Data assimilation and parameter estimation via ensemble Kalman filter 
coupled with stochastic moment equations of transient groundwater flow, Water Resour. Res., 49, 1334-1344, 2013.

Panzeri, M., Riva, M., Guadagnini, A., and Neuman, S. P.: Comparison of Ensemble Kalman Filter Groundwater-Data Assimilation Methods Based on Stochastic Moment Equations and Monte Carlo Simulation, Adv. Water Resour., 66, 8-18, 2014.

Panzeri, M., Riva, M., Guadagnini, A., and Neuman, S. P.: EnKF coupled with groundwater flow moment equations applied to Lauswiesen aquifer, Germany, J. Hydrol., 521, 205-16, 2015.

Phale, H. A. and Oliver, D. S.: Data Assimilation Using the Constrained Ensemble Kalman Filter, Soc. Petrol. Eng., 16, 331-342, 2011.

Post, V. E. and von Asmuth, J. R.: Review: Hydraulic head measurements-new technologies, classic pitfalls, Hydrogeol. J., 21, 737-750, 2013.

Reichle, R. H., McLaughlin, D. B., and Entekhabi, D.: Hydrologic data assimilation with the ensemble Kalman filter, Mon. Weather Rev., 130, 103-114, 2002.

Robert, C.: The Bayesian Choice: From Decision-Theoretic Foundations to Computational Implementation, Springer Science \& Business Media, New York, 2007.

Samuel, J., Coulibaly, P., Dumedah, G., and Moradkhani, H.: Assessing Model State and Forecasts Variation in Hydrologic Data Assimilation, J. Hydrol., 513, 127-141, 2014.

Tian, X., Xie, Z., and Dai, A.: A land surface soil moisture data assimilation system based on the dual-UKF method and the Community Land Model, J. Geophys. Res., 113, D14127, doi:10.1029/2007JD009650, 2008.
Valstar, J. R., McLaughlin, D. B., Te Stroet, C., and van Geer, F. C.: A representer-based inverse method for groundwater flow and transport applications, Water Resour. Res., 40, W05116, , doi:10.1029/2003WR002922, 2004.

Vrugt, J. A., Gupta, H. V., Bouten, W., and Sorooshian, S.: A Shuffled Complex Evolution Metropolis algorithm for optimization and uncertainty assessment of hydrologic model parameters, Water Resour. Res., 39, 1201, doi:10.1029/2002WR001642, 2003.

Vrugt, J. A., Gupta, H. V., Nualláin, B., and Bouten, W.: Real-time data assimilation for operational ensemble streamflow forecasting, J. Hydrometeorol., 7, 548-565, 2006.

Wan, E. A., Van Der Merwe, R., and Nelson, A. T.: Dual Estimation and the Unscented Transformation., in: NIPS, pp. 666-672, Citeseer, 1999.

Wen, X. H. and Chen, W. H.: Real-time reservoir updating using ensemble Kalman Filter: The confirming approach, Soc. Petrol. Eng., 11, 431-442, 2007.

Zhou, H., Gómez-Hernández, J. J., Hendricks Franssen, H.-J., and Li, L.: An approach to handling non-Gaussianity of parameters and state variables in ensemble Kalman filtering, Adv. Water Resour., 34, 844-864, 2011.

Zhou, H., Gómez-Hernández, J. J., and Li, L.: Inverse methods in hydrogeology: evolution and recent trends, Adv. Water Resour., 63, 22-37, 2014. 